\title{
A Multi-chamber Model of Combustion Instabilities and its Assessment using Kilohertz Laser Diagnostics in a Gas Turbine Model Combustor
}

\author{
YunTao Chen*, James F. Driscoll \\ Department of Aerospace Engineering, University of Michigan, Ann Arbor, MI 48109
}

\begin{abstract}
A multi-chamber model for the combustion instabilities manifested in a gas turbine model combustor was developed. The proposed model was used to explain the dependencies of instability frequency on burner geometry and other flow parameters, some of which could not be reconciled with previous models. The new model was built upon the Helmholtz analysis of two connected resonators. The instability frequency as well as the complex pressure ratio between two chambers were predicted by solving ordinary differential equations. To assess the assumptions and predictions of the proposed model, the spectra and magnitude of the oscillations of pressure, heat release rate, and velocity were measured for four different operating conditions: rich (R1), lean (L1), stoichiometric (S1), and reduced flow rate (R2) with a kilohertz laser diagnostic system. These measurements reconfirmed that the instability is of Helmholtz type. A global equivalence ratio that is consistently greater than unity was identified to be an enabling factor for combustion instability. This is also in agreement with the predictions made by the proposed model. Furthermore, the model was shown to be able to predict the right trend of instability frequency when multiple parameters were changed. It is concluded that the current model is an improvement over previous models, because the acoustic coupling between different chambers of the burner was considered.

Keywords: Combustion instability, reduced-oder modeling, gas turbine combustion, partially premixed combustion
\end{abstract}

\section{Introduction}

\subsection{Combustion instability in gas turbine engines}

Combustion instability is characterized by the large oscillations of heat release and flame location, coupled with an exponentially growing pressure oscillation amplitude or a limit cycle of pressure oscillations sustained at a large amplitude. Instabilities represent a major obstacle to implementation of low- $\mathrm{NO}_{\mathbf{x}}$ lean-premixed technology in gas turbine engines, and they may lead to the catastrophic failure of the engine components. The relationship between pressure and heat release osciallation was investigated by Lord Rayleigh [1], who stated that acoustic oscillations are amplified by heat release when the latter is in phase with pressure oscillations. This is called the Rayleigh criterion. There are many causes for the oscillations, Zinn and Lieuwen [2] and Mongia et al. [3] explained that anchoring a premixed flame within an engine can be difficult. Heat release oscillations can be caused by equivalence ration oscillations [4] as well as flame surface area oscillations [5].

\footnotetext{
${ }^{*}$ Corresponding author, current affiliation: Siemens Energy Inc., Orlando, FL, USA

Email address: yuntaoc@umich.edu (YunTao Chen)
} 
$v \quad$ Radial velocity

\section{Greek Symbols}

$\Delta h_{\mathrm{f}}^{\circ} \quad$ Lower heating value

$\omega \quad$ System instability frequency

$\omega_{0} \quad$ Plenum Helmholtz frequency

$\omega_{2} \quad$ Combustion chamber Helmholtz frequency

$\Phi \quad$ Equivalence ratio

$\psi_{20} \quad$ Combust. chamber/plenum phase difference

$\tau_{\mathrm{c}} \quad$ Convection time delay

$\zeta \quad$ Damping ratio

Mathematical Symbols

$\dot{x} \quad$ Rate of variable $x$

$|x| \quad$ Magnitude of complex number $x$

$\bar{x} \quad$ Average of variable $x$

$x^{\prime} \quad$ Fluctuation of variable $x$

Subscripts

$0 \quad$ Plenum properties

1 Injector properties

2 Combustion chamber properties

3 Exhaust duct properties

This work focuses on geometries that can lead to a Helmholtz-type instability. It is noted that within a full-scale engine, instability can be caused by standing waves, a Helmholtz resonance, or by a complex acoustic wave pattern. The Helmholtz resonance occurs when a relatively short volume of fluid is connected to a small orifice. For example, Krebs et al. [6] state that in Siemens engines Helmholtz resonances are called "breathing modes" and may occur in the $100-400 \mathrm{~Hz}$ range. Full-scale injector studies at Pratt and Whitney by Cohen and Banaszuk [7] and by Cohen et al. [8] found that their instability was due to a Helmholtz resonance at $200 \mathrm{~Hz}$. Previous laboratory-scale experiments also have shown that a Helmholtz resonance occurs when a combustion instability is detected; these findings were reported by Temme et al. [9], Zähringer et al. [10], Uhm and Acharya [11], and Durox et al. [12]. General reviews of the many possible types of instabilities are given by McManus et al. [13], Ducruix et al. [14], Zinn and Lieuwen [2], and Mongia et al. [3].

The combustion chamber of a gas turbine engine can be considered to be a series of connected chambers, as 
shown in figure 1a. Just downstream of the combustor is an exit orifice that leads to the first stage of the turbine. Figure 1b illustrates two chambers and two orifices. Based on this simplifying idea, the DLR (German Aerospace Center) Gas Turbine Model Combustor (GTMC) was designed by Meier and colleagues at DLR Stuttgart [15]. As shown in figure 2, the GTMC features a ring of fuel injectors surrounded by dual swirling air injectors, in a plenum-injector-combustor-exhaust configuration. It contains the basic feature of a practical combustor within a geometrically simple and optically accessible setup. The experimental measurements and reduced order modeling of combustion instabilities in this work will be based on the GTMC.

\subsection{Previous research on the GTMC}

Published literature of investigations on GTMC date back as far as 2003 [16, 17]. Meier and colleagues made extensive imaging measurements in their GTMC [15, 18, 19, 20, 21, 22, 23, 24, 25]. One of their main interests was the flame-flow interaction. In particular, they studied how the vortex-like motion of the helical Precessing Vortex Core (PVC) interacts with the flame. They found that the PVC-flame interaction occurs in both cases: when an instability is present $[18,19,20,21]$ as well as when it is absent $[15,22,23,24,25]$. They imaged the flame using kilohertz OH Planar Laser Induced Fluorescence (PLIF) and the PVC with kilohertz Particle Image Velocimetry (PIV). They found that the PVC rotation causes flow and flame oscillations at frequencies of 400 500 $\mathrm{Hz}$, while the pressure oscillations associated with the combustion instability occur at a lower frequency around $300 \mathrm{~Hz}$. The fact that the PVC rotation and the combustion instability occur at different frequencies causes complications in the understanding of the physical mechanism of the heat release. They showed that the PVC-flame interaction affects where the heat is released, and it affects the time delay between pressure oscillations and the heat release rate oscillations.

Steinberg et al. $[18,20]$ found that the pressure fluctuations in the plenum of GTMC are $60^{\circ} \sim 80^{\circ}$ out of phase with those in the combustion chamber and they inferred that the acoustic resonance is of the Helmholtz type. Their measured phase difference is reasonable for a Helmholtz resonator and is likely influenced by acoustic damping at the injector. Their results indicate that the pressure field and the acoustic resonance may be relatively simple, as the Helmholtz resonator has uniform pressure fluctuations. However the heat release that drives the instability may be complex and due to PVC-flame interactions.

Allison et al. [26] operated a GTMC burner at Michigan that is identical to the DLR design. They investigated the effect of varying geometry parameters, flow rate, and equivalence ratio for various fuels. The measured frequency decreased with an increase in plenum volume and exhaust tube length, and increased with increasing mass flow rates for different fuels. The fact that instability frequency depends on both the geometries of plenum and exhaust duct indicates that the observed combustion instability is caused by a multi-chamber resonance.

In later works, Allison et al. investigated a DME flame [27, 28]. The results show that DME flames behave similar to other fuels in terms of dependency of frequency on equivalence ratio and mass flow rate. The instability frequencies of different fuels are shown to be proportional to the air mass flow rate. Another important aspect of this later work is that they observed a stable flame at $\Phi=0.75$ and an unstable flame at $\Phi=1.2$. This is detailed in table 1. At $\Phi=0.75$ the pressure oscillation amplitude is so small that an accurate determination of instability was not possible. 


\subsection{Previous models of combustion instability}

Combustion instability encountered in the GTMC is caused by the interactions between turbulent combustion, fluid dynamics, and acoustics. Each of these processes are challenging to model, hence modeling combustion instability in the GTMC with a high fidelity model is particularly difficult. See and Ihme [29] used the Flamelet Progress Variable (FPV) model in the context of Large Eddy Simulation to model the GTMC at stable operating conditions.

In view of the obstacles in using the high fidelity models, another approach to model combustion instability has been developed. This approach is to use reduced order models to represent the basic physics and neglect the high order effects. Such an approach has been taken by several research groups with various experimental geometries. Notably, Hathout et al. [30] studied the case where a combustion chamber with an inlet and an outlet acts as a Helmholtz resonator. Heat release rate of the combustion chamber was related to flame surface area, which was determined by local flame speed and inlet velocity. Pressure and velocity were correlated in the inlet pipe through conservation of momentum. In this way a second-order ordinary differential equation was derived for the pressure fluctuations. Through stability analysis of the equation, the stable operating envelop was determined.

Researchers from Laboratoire EM2C at École Centrale Paris studied other geometries [31, 32]. Specifically, Schuller et al. [33] studied the acoustic coupling effects of a plenum-injector-combustor system using a reduced order model through matrix eigenvalue analysis. Palies et al. [34] studied the same setup with fluctuating heat release rate within the Flame Describing Function (FDF) framework. Furthermore, this analysis was compared to a Helmholtz solver by Silva et al. [35], with good agreement.

\subsection{Motivation and outline of this work}

Despite the many previous studies, there are several unanswered questions about the combustion instability in the GTMC that need to be addressed. For example, an explanation is needed to understand the dependence of instability frequency on burner geometries and mass flow rates as observed in the work of Allison et al. [26, 27]. The previous model by Hathout et al. is a useful concept but it is not adequate for the present geometry because it only considers one volume (the combustion chamber). To develop a new model to provide the necessary explanation, further experimental measurements on the frequency spectra of velocity, heat release, and pressure oscillations are needed.

Therefore, the scope of the current work is to perform a set of diagnostic experiments to extract information relevant to thermo-acoustic instabilities in the GTMC at different operating conditions, then to develop a reduced order model based on the experimental observations to provide an explanation to current and previous observations. 
The proposed model is based on concepts in the Hathout et al. [30] model and the model of Schuller et al. [33], but it is extended to consider heat release in one chamber and no heat release in a second, coupled chamber.

The structure of the paper is the following: first the experimental setup is introduced, and the results from our experimental measurements are discussed. This is followed by the introduction of the proposed reduced order model. Then comparison of the predictions from the proposed reduced order model with experimental observations are provided, and conclusions are drawn.

\section{Experimental characterizations of combustion instability in the GTMC}

As aforementioned, more information regarding the relationship between combustion and fluid dynamics inside the combustion chamber is needed to aid the development of a more representative model of combustion instability inside GTMC. In this section, the experimental configuration of current study is presented. And the results of the high speed laser diagnostics are discussed.

\subsection{Experimental setup}

A schematic drawing of the platform of current study, the GTMC, is shown in figure 2. The injector of the GTMC consists of a central air nozzle, an annular fuel nozzle, and a co-annular air nozzle. Both air nozzles supply swirling dry air at atmospheric pressure and temperature from a common plenum. The inner air nozzle has an outer diameter of $15 \mathrm{~mm}$. The outer annular nozzle has an inner diameter of $17 \mathrm{~mm}$ and an outer diameter of $25 \mathrm{~mm}$. The measured swirl number is approximately 0.55. Non-swirling fuel is provided through three exterior ports into the annular nozzle which is subdivided into 72 channels of dimension $0.5 \mathrm{~mm} \times 0.5 \mathrm{~mm}$. The width of the fuel annulus is less than $0.5 \mathrm{~mm}$. The exit plane of the central air nozzle and fuel nozzle lies $4.5 \mathrm{~mm}$ below the exit plane of the outer air annulus. The exit plane of the outer air annulus will be referred to as the burner surface. The combustion chamber has a square cross section of $85 \mathrm{~mm}$ in width and $110 \mathrm{~mm}$ in height. The exit of the burner has a tapered lid which leads to an exhaust duct with a diameter of $40 \mathrm{~mm}$ and a height of $50 \mathrm{~mm}$. The combustion chamber is enclosed with fused silica windows, whose thickness is $1.5 \mathrm{~mm}$, for flame visualization. The burner was operated with dimethyl ether (DME) fuel. An external cylindrical chamber was used for the equal division of the fuel flow into three separate lines which lead to the fuel ports on the burner. Mass flow rates for air and fuel lines were controlled by sonically choked orifices.

Pressure measurements are made using piezoelectric microphones (PCB 378C10) mounted on the plenum of the GTMC. From previous measurements $[20,36]$ we know that the combustion chamber pressure fluctuation is at the same frequency as that of the plenum, but with a phase lag. Hence the combustion chamber pressure information can be obtained from plenum pressure measurements without obstructing the optical measurements. The pressure signal is relaid by a signal conditioner (PCB 482C05) to an oscilloscope (LeCroy Waverunner 6100A) at $1 \mathrm{MHz}$. When combustion chamber pressure needs to be measured, one glass wall of the chamber is replaced with a steel plate where pressure ports were drilled. For details of this measurement, please refer to appendix B.

The optical diagnostics consist of kilo-hertz PIV, formaldehyde PLIF, and $\mathrm{CH}^{*}$ chemiluminescence measurements. The interrogation windows of PIV is positioned such that it can be used to resolve the spectrum of the injector jet velocity both axially and radially. $\mathrm{CH}^{*}$ chemiluminescence is used as a indicator for the integrated global heat 
release rate. The formaldehyde PLIF is used to visualize the instantaneous heat release/flame structure. Following a method extended from that of Allison et al.[28], the sharp edges of the formaldehyde PLIF signal are identified as flame fronts. Then the flame surface area (FSA) inside the PLIF field of view is calculated. After taking the temporal and spatial average of FSA in each frame, the flame surface density (FSD), which is a two dimensional representation of the probability of the existence of flame in a given region, can be deduced.

FSA and FSD are not only the visualization of the instantaneous and aggregate flame structure on the plane of the laser sheet. In certain conditions, they have also been related to the local heat release rate. Rosenberg and Driscoll [37] measured flame index in this GTMC experiment and found that over $80 \%$ of the flamelets are of the premixed type, since extensive fuel-air mixing occurs upstream of the lifted flame base. For premixed flames, there is a strong correlation between heat release rate and flame surface density [38, 39, 40]. For non-premixed flames, flame surface density has been successfully used as an indicator of heat release by Steinberg et al. [18, 20]. Therefore, whereas $\mathrm{CH}^{*}$ chemiluminescence provides spatially integrated total heat release rate information, FSA and FSD derived from formaldehyde PLIF can provide the local heat release distribution. The comparison of these two heat release rate imaging methods will be discussed in a separate work.

The PIV system employs $532 \mathrm{~nm}$ beam generated from the high speed diode-pumped Nd:YAG laser while the PLIF system uses a $355 \mathrm{~nm}$ beam from a similar laser. The field of view is $15 \mathrm{~mm} \times 15 \mathrm{~mm}$ for the PIV system and is $20 \mathrm{~mm} \times 40 \mathrm{~mm}$ for the PLIF system. The PIV interrogation box is $64 \times 64$ pixels $(2.5 \mathrm{~mm} \times 2.5 \mathrm{~mm})$. The field of view for $\mathrm{CH}^{*}$ chemiluminescence measurement is $40 \mathrm{~mm} \times 80 \mathrm{~mm}$. The relative locations of these interrogation windows are shown in figure 2. Additional information regarding the laser diagnostics can be found in appendix A.

As shown in table 2, four test cases are considered in the present study. At the air flow rate of $282 \mathrm{~g} / \mathrm{min}$ (which corresponds to case "B" in DLR experiments [15]), three of the cases are: R1 (R:rich), S1 (S:stoichiometric), and L1 (L:lean) with equivalence ratio of 1.2, 1.0, and 0.75 respectively. At a reduced flow rate of $226 \mathrm{~g} / \mathrm{min}(80 \%$ of standard case), the experiment was repeated for the rich case (R2).

Table 2: Test case matrix

\begin{tabular}{ccccc}
\hline Case & $\Phi$ & $\dot{m}_{\mathrm{a}}[\mathrm{g} / \mathrm{min}]$ & Fuel & Instability \\
\hline \hline R1 & 1.2 & 282 & DME & Strong \\
S1 & 1.0 & 282 & DME & Intermitent \\
L1 & 0.75 & 282 & DME & None \\
R2 & 1.2 & 226 & DME & Weak \\
\hline
\end{tabular}

The test case matrix is designed in such a way to: $i$ ) explore the differences in spatial and temporal structure of a unstable (R1) and a stable (L1) flame; ii) investigate the intermittent instability case at stoichiometric condition (S1); iii) explore the effect of reducing mass flow rate (between R1 and R2).

Each run for a certain case lasts 1 second. Pressure data was recorded at $1 \mathrm{MHz}$. PIV and PLIF data were obtained at $4 \mathrm{kHz}$, while the $\mathrm{CH}^{*}$ chemiluminescence data had a sampling rate of $20 \mathrm{kHz}$. The pressure magnitude, velocity magnitudes, total flame surface area, and total count of $\mathrm{CH}^{*}$ chemiluminescence signal were assembled as time series data. Fast Fourier Transform (FFT) analysis was performed on those temporal domain data and the spectra of these parameters were obtained. No spectral filtering/smoothing was applied in the spectral domain, and 
the resolution of the spectra of all quantities is $1 \mathrm{~Hz}$.

\subsection{Experimental results}

Thermo-acoustic instability is characterized by a large pressure fluctuation in the combustion system. Thus the acoustic properties of the system are important and were investigated.

The pressure amplification factor in the GTMC was measured with a loudspeaker sweeping through a range of input frequencies. The plenum and combustion chamber were separated and the response to this external source of perturbation was measured separately. In each case, the loudspeaker was placed right at the outlet of the component (plenum or combustion chamber) so that the maximum amount of emitted acoustic power can be recorded. The results are presented in figure 3 .

Figure 3 shows the amplification factor of pressure wave in plenum and combustion chamber. This factor is the ratio of the measured sound level inside the plenum or combustion chamber divided by the sound level measured close to the loudspeaker. In this way, the bias of speaker output in the spectral domain can be eliminated. The spectrum of acoustic response of plenum (solid blue, top curve) has several prominent features. One cluster of peaks is near $300 \sim 350 \mathrm{~Hz}$, and the other one is in the $600 \sim 800 \mathrm{~Hz}$ range. The acoustic response of combustion chamber is much lower than that of the plenum. This is caused by the damping effect of the glass walls of the combustion chamber. The amplification factor for the combustion chamber (dashed blue, bottom curve) is consistently below 1 . This means the sound level measured at the chamber is smaller than the input acoustic wave, thus the lower curve in figure 3 indicated the degree of damping in the combustion chamber.

The acoustic response was also measured when air was flowed through the system (with plenum and combustion chamber properly assembled) at normal flow rate $(282 \mathrm{~g} / \mathrm{min})$ without fuel injection. The noise generated by the air flow served as the input source and the response of the system was measured in the plenum and the combustion chamber. The results are shown in figure 4. The returned signal by this method is on average 10 times stronger than the previous speaker-excitation method. In this figure, a dispersed but prominent peak in the plenum pressure PSD (solid line in figure 4) in the range of 700 800 Hz is observed. Similar features in this range was observed in figure 3. However the other peak at $300 \sim 350 \mathrm{~Hz}$ in figure 3 is not observed here.

Similar to the speaker-excitation method, the pressure oscillation amplitude in the combustion chamber is much smaller than that inside the plenum (dashed line in figure 4). No clear peak like the one in the plenum was observed. However, several prominent features in the range of $200 \mathrm{~Hz}$ to $400 \mathrm{~Hz}$ were noticeable. Due to their low strength, no conclusions were made on the nature of these peaks, but it may indicate that certain acoustic features between 200 $\mathrm{Hz}$ and $400 \mathrm{~Hz}$ exist in the system. Similar features were also observed in figure 3.

The GTMC was then operated with the fuel added and ignited according to the test matrix specified in table 2. The spectra of plenum pressure, $\mathrm{CH}^{*}$ chemiluminescence, $\mathrm{CH} 2 \mathrm{O}$ PLIF signal and velocities were measured and presented in figure 5 . The first row of figure 5 shows the power spectra of all four cases, normalized by a reference power in arbitrary units. It shows that the difference between a unstable case (R1) and a stable case (L1) is very distinct. Whereas $\mathrm{R} 1$ has a single peak in $310 \sim 320 \mathrm{~Hz}$ range, L1 has no visible prominent peak. The spectrum of $\mathrm{S} 1$ (stoichiometric) is similar to that of R1, with a peak at the thermo-acoustic instability frequency of $310 \mathrm{~Hz}$ at a smaller magnitude. The single peak in R2 is smaller than R1, its frequency also shift to about $290 \mathrm{~Hz}$. This means 
that as mass flow rates decreases, the instability becomes weaker and shifts to a lower frequency. Note that the pressure (as well as chemiluminescence and FSA) signal of S1 and R2 are amplified 5 times to be shown on the same scale as R1.

The spectra of integrated $\mathrm{CH}^{*}$ chemiluminescence, indicating the total heat release rate, is presented in the second row of figure 5. The $\mathrm{CH}^{*}$ chemiluminescence spectra are almost identical to those of the pressure, showing that pressure and heat release fluctuations are indeed closely coupled. Note that a very slight peak frequency shift between the pressure and the $\mathrm{CH}^{*}$ chemiluminescence spectra exists for case $\mathrm{R} 2$. The reason was that the two signal were taken at two separate runs. The pressure signal shown here was taken simultaneously with the PLIF signal shown in the third row. It was known that the instability frequency has a dependency over the burner surface temperature, and care was taken to record data at the same burner surface temperature as indicated by an embedded thermal couple. However, occasionally between data campaigns such frequency shift within a $10 \mathrm{~Hz}$ range still exists for the same operating condition. The spectrum of the $\mathrm{CH}^{*}$ chemiluminescence was checked with the corresponding pressure signal spectrum, and within the $1 \mathrm{~Hz}$ precision of our spectral analysis the two match exactly.

In third row of figure 5 the power spectra of the total flame surface area (FSA) fluctuations as identified by our PLIF edge detection algorithm are presented. In the case of R1, FSA fluctuates at a single frequency that is the same as the pressure. No spike is observed in the pressure and heat release spectra of L1. But the flame surface area has a very strong peak at $420 \mathrm{~Hz}$. This is caused by the helical PVC that is rotating around the swirler. This flow feature has been well characterized in literature [19, 20]. As the PVC rotates in the combustion chamber, it takes reactants and products with it. Hence there will be a overall fluctuation of formaldehyde PLIF signal strength in the frame. However, this local phenomenon has no effect over the total heat release rate since there is no spectral peak around $420 \mathrm{~Hz}$ found in the corresponding $\mathrm{CH}^{*}$ chemiluminescence spectrum. The effect of the PVC is also evident in S1, albeit with a smaller magnitude. R2 on the other hand only shows a single peak at the themo-acoustic instability frequency of $290 \mathrm{~Hz}$. This is similar to R1, only at a smaller magnitude.

As shown in figure 6, the information of flame edges as identified from formaldehyde PLIF signal can also be translated into flame surface density (FSD), which is a spatial representation of the probability of flame existence in the PLIF laser plane. The spatial distributions of FSD in R1 and L1 are quite different. The flame in R1 is distributed and flat, near the burner surface. The flame in L1 is more lifted, showing a "V" shape.

A FFT analysis was carried out to explore the frequency spectrum of both axial and radial velocity components, as shown in fourth and fifth rows of figure 5 . The fourth row shows the power spectrum of radial velocity for all cases. There are two peaks in $\mathrm{R} 1$ radial velocity spectrum, which correspond to the thermo-acoustic instability frequency $(\sim 320 \mathrm{~Hz})$ and PVC frequency $(\sim 460 \mathrm{~Hz})$. This shows that the pressure fluctuation in the unstable case affects the velocity fluctuations. In L1 we only have one peak for the PVC above $400 \mathrm{~Hz}$ because of the absence of combustion instability. This PVC frequency is also the frequency of the peak in flame surface area spectrum of L1 in the third row of figure 5. This supports our claim that the observed peak in figure 5 for L1 is an local phenomenon affected by the PVC. The radial velocity fluctuations in S1 are stronger than R1 and L1 at both the instability frequency and the PVC frequency. This may be explained by the fact that the velocity fluctuations are large during the frequent flame re-position when it switches between the stable mode and unstable mode. Lastly, the spectrum of R2 radial velocity resembles that of $\mathrm{R} 1$ with a much smaller magnitude. 


\subsection{Discussion of experimental results}

The experimental investigation above encompasses pressure, velocity, and heat release (with flame surface and chemiluminescence as proxies) measurements for four different operating conditions. A wide range of differences between each case have been observed, which lead to the following findings.

i) The investigation of the system acoustics without flame reveals that certain acoustic modes exist in the system. Specifically, from the speaker-excitation method, we see that the plenum has inherent mode in $300 \sim 350 \mathrm{~Hz}$ as well as $600 \sim 800 \mathrm{~Hz}$. According to previous calculation, the $300 \sim 350 \mathrm{~Hz}$ mode should correspond to the plenum Helmholtz mode. The nature of the $600 \sim 800 \mathrm{~Hz}$ mode is unclear. The acoustic properties of the combustion chamber was not clearly determined because the glass walls were too dissipative and the excitation sources were not strong enough to excite any of its modes. With the air-flow method, the system mode in near $800 \mathrm{~Hz}$ was excited. This might be from the mode of plenum in the same frequency range. The $300 \sim 350 \mathrm{~Hz}$ mode, which is linked to the plenum Helmholtz mode, was not excited by the air-flow method.

ii) The PLIF data provides spatially-resolved contours of the flame surface density, which are indicators of the contours of local flame distribution. However, the FSA and FSD obtained from PLIF need to be used with caution. Because they are obtained only from one plane in the flame, they are prone to the influences of local flow features such as the PVC. Nonetheless, both formaldehyde PLIF and $\mathrm{CH}^{*}$ data show that the spectrum of heat release rate oscillations has a sharp spike at the same frequency as the spike in the pressure spectrum for unstable cases. This proves that the heat release rate is coupled to the pressure fluctuations. The kilohertz PIV data show that 
the velocity field also has a peak at the instability frequency in unstable flames. The coincidence of all oscillation frequencies supports the assertion that a Helmholtz mode is present in the system when it is unstable.

iii) From previous studies by Allison et al. [27] it is known that pressure fluctuation magnitude and flame surface density distribution are different between an unstable (R1) an stable (L1) flame. Velocity spectrum analysis in this study reveals that the effect of combustion instability would change the axial and radial velocity distribution as well. In an unstable flame the major dynamics happens in the radial direction, with velocity undergoing a large oscillation radially. In a stable flame the velocity oscillation is more evenly distributed between axial and radial directions. Considering that the flame in an unstable case sits in the outer recirculation zone, it is the radial velocity component of the swirler jet that is being "pushed" by the flame back and forth. We do not know if the velocity re-distribution between radial and axial components is caused by heat release or if it controls heat release, but we do find the a link between velocity and heat release.

iv) The spectra in figure 5 provide insight on the relationship between the PVC and combustion instability. As summarized in table 3, the PVC is present in both R1 and L1 flames. In flame R2, we do not see evidences of the PVC, but we still saw a peak in the pressure oscillation. Additionally, the increase of PVC frequency from L1 to S1 to $\mathrm{R} 1$ follows the increase of total flow rate closely. This means that the PVC is more influenced by flow parameters than the existence of combustion instability. While instability and PVC do work together in determining the velocity distribution, the spectral data show that the presence of a PVC is not required for a combustion instability.

$v$ ) The pressure and chemiluminescence spectra of S1 (intermittent instability) and R2 (weak instability) are very similar, but they represent two very different phenomena. Case S1 is a transitional state that can be thought of as a temporal average of R1 and L1. At a lower flow rate, the instability frequency of R2 is lower. From flame surface density in figure 6 we see that the flame is more lifted off and less intense. The velocity magnitude fluctuation is also fairly small for case R2 as compared to R1, L1, or S1. This leads us to believe that a reduced flow rate makes the flame "milder" even though the equivalence ratio still determines the characteristics of the instability.

\section{Reduced Order Modeling of Combustion Instability in the GTMC}

To establish a conceptual framework to understand the combustion instability phenomena observed in the GTMC, a reduced order model is proposed. In this section, the derivation of this model will be presented.

It has been concluded that the combustion instability in the GTMC is dominated by a Helmholtz mode (detailed in appendix B). Hence our proposed reduced order model is based on a Helmholtz resonator analysis. Similar analysis has also been carried out previously on several different setups[30, 33, 34]. The proposed model extended the single chamber analysis done by Hathout et al. [30] and coupled it with the multi-chamber analysis done by Schuller and Palies et al. $[33,34]$. It is based on two ordinary differential equations, with assumed functions for heat release and acoustic damping. Compared to these studies, the proposed model has the advantage of being able to analyze a multi-chamber system without the need for a experimentally determined Flame Describing Function (FDF).

In the proposed model, the GMTC is simplified and takes the form of an abstract system consisting of four connected bodies, as shown in figure 7. Here the four major elements of the system correspond to (0) plenum, (1) injector/swirler, (2) combustion chamber, and (3) exhaust duct. The key assumptions of this model are: $i$ ) the system elements are assumed to be zero dimensional in space. That is, all gas properties (pressure, temperature, 
etc.) are uniform within each element; $i i)$ the system is operated at low Mach numbers, no compressibility effect is considered. With these assumptions, each element is characterized by its length and cross-section area. Cold mixture flows from the plenum through the injector into the combustion chamber. It is then immediately ignited and exits from the exhaust duct. The complex flow field within the injector is neglected.

The plenum and combustion chamber are identified as the Helmholtz volumes. The foundation of the present model is the single chamber Helmholtz analysis extended from the work by Hathout et al. [30]. This analysis is detailed in appendix C. In the next sections, we apply the analysis to both plenum and the combustion chamber of the GTMC to obtain the governing equations of the proposed model.

\subsection{Plenum pressure equation}

The governing equation for the pressure fluctuation inside a a single chamber Helmholtz resonator is Eq. C.13 in appendix C. Because only cold air flows through the plenum, the effect of variations in heat capacity, heat loss, and heat release is neglected. Additionally, because the upstream of the inlet pipe to the plenum is long and narrow, conditions upstream of the plenum are neglected. With these conditions, we can write Eq.(C.13) with the notation in the GTMC framework $(\{\mathrm{v}, \mathrm{e}, \mathrm{d}\} \rightarrow\{0,1,2\})$ :

$$
\frac{d^{2} P_{0}^{\prime}}{d t^{2}}+\left(\frac{C_{0}^{2} A_{1}}{V_{0} l_{1}}\right) \cdot P_{0}^{\prime}=\left(\frac{C_{0}^{2} A_{1}}{V_{0} l_{1}}\right) \cdot P_{2}^{\prime} .
$$

The meaning of the symbols can be found in the nomenclature. The Helmholtz characteristic frequency $\omega_{0}$ of the plenum is defined to be

$$
\omega_{0}=\sqrt{\frac{C_{0}^{2} A_{1}}{V_{0} l_{1}}},
$$

then Eq.(1) can be written as:

$$
\frac{d^{2} P_{0}^{\prime}}{d t^{2}}+\omega_{0}^{2} \cdot P_{0}^{\prime}=\omega_{0}^{2} \cdot P_{2}^{\prime}
$$

\subsection{Combustion chamber pressure equation}

Combustion chamber inlet temperature $(\sim 300 \mathrm{~K})$ is very different from that inside the chamber $(\sim 2000 \mathrm{~K})$, hence the effect of the difference in heat capacities has to be considered. The glass walls of the chamber is thin so the heat loss across them is not negligible.

With these conditions, Eq.(C.13) can be written as:

$$
\frac{d^{2} P_{2}^{\prime}}{d t^{2}}+\mathscr{T}_{\mathrm{ii}} \frac{d P_{2}^{\prime}}{d t}+\left(\mathscr{T}_{\mathrm{iii}}+\mathscr{T}_{\mathrm{iv}}\right) P_{2}^{\prime}=\frac{\gamma_{2}-1}{V_{2}} \frac{d \dot{Q}_{2}}{d t},
$$

with

$$
\begin{gathered}
\mathscr{T}_{\mathrm{ii}}=\frac{\gamma_{2}-1}{V_{2}} \frac{1}{\Omega \rho_{2} R_{2}}, \\
\mathscr{T}_{\text {iii }}+\mathscr{T}_{\mathrm{iv}}=\frac{\gamma_{2}-1}{\gamma_{0}-1}\left(\frac{C_{0}^{2} A_{1}}{V_{2} l_{1}}\right)+\left(\frac{C_{2}^{2} A_{3}}{V_{2} l_{3}}\right) .
\end{gathered}
$$

Here $\Omega$ is the thermal resistance for heat transfer and $R$ is the gas constant. If we define the combined Helmholtz resonance frequency of combustion chamber as:

$$
\omega_{2}=\sqrt{\frac{\gamma_{2}-1}{\gamma_{0}-1}\left(\frac{C_{0}^{2} A_{1}}{V_{2} l_{1}}\right)+\left(\frac{C_{2}^{2} A_{3}}{V_{2} l_{3}}\right)},
$$


then Eq.(4) can be written as:

$$
\frac{d^{2} P_{2}^{\prime}}{d t^{2}}+2 \zeta_{2} \cdot \omega_{2} \cdot \frac{d P_{2}^{\prime}}{d t}+\omega_{2}{ }^{2} \cdot P_{2}^{\prime}=\frac{\gamma_{2}-1}{V_{2}} \cdot \frac{d \dot{Q}^{\prime}}{d t},
$$

with

$$
\zeta_{2}=\frac{\gamma_{2}-1}{V_{2}} \frac{1}{2 \omega_{2} \Omega \rho_{2} R_{2}}
$$

being the damping coefficient of the combustion chamber.

\subsection{Wall heat loss modeling}

As shown in Eq.(7), the damping coefficient $\zeta_{2}$ is proportional to the heat loss across the wall and is inversely proportional to the thermal resistance $\Omega$.

Here we consider the heat transfer from the hot reacting fluid inside the combustion chamber into the ambience. The glass walls are the intermediate layers between the heat source (flame) and the heat sink (ambience). There are two paths of heat transfer. Through the first path heat convects from fluid to the wall, then conducts across the wall and passes into the ambience through natural convection (the ambience is quiescent). Through the second path heat radiates directly from combustion products into the ambience. This is because the fused silica windows can be considered as transparent for electromagnetic waves with a wavelength between $200 \mathrm{~nm}$ and $4 \mu \mathrm{m}$ [41]. For a black body radiation source of $2000 \mathrm{~K}, 85 \%$ of its radiated power is at wavelengths below $4 \mu \mathrm{m}$. Hence the inference of the glass walls on the radiative heat transfer is small and is neglected. The thermal resistance of these two paths can be separately calculated. The details of this heat transfer analysis can be seen in appendix D.

\subsection{Heat release modeling}

The solution of Eq.(6) requires the relationship between heat release rate fluctuation and pressure fluctuation. First we make the assumptions that: $i$ ) the chemistry involved is a one-step reaction, there is no intermediate step or partial fuel decomposition; ii) all chemical reactions are much faster compared to the flow residence time (Damköhler number is infinite); iii) heat release is not affected by the mean flow rate or turbulence levels, for example blow-out is not considered; and $i v$ ) the incoming fuel flow rate is constant and there is no fuel-trapping near the injection nozzle due to complex flow interactions. Then the total heat release rate $\dot{Q}$ can be related to the total mass flow rate $\left(\dot{m}_{\text {tot }}=\dot{m}_{\mathrm{f}}+\dot{m}_{\mathrm{a}}\right)$ and lower heating value $\left(\Delta h_{\mathrm{g}}^{\circ}\right)$ of the fuel-air mixture by:

$$
\dot{Q}=\frac{\Delta h_{\mathrm{g}}^{\circ}}{A F R_{\mathrm{st}}} \cdot \min \left(\dot{m}_{\mathrm{f}} \cdot A F R_{\mathrm{st}}, \dot{m}_{\mathrm{a}}\right),
$$

where equivalence ratio $\Phi$ and stoichiometric air-fuel ratio $\left(A F R_{\mathrm{st}}\right)$ are related to fuel flow rate $\left(\dot{m}_{\mathrm{f}}\right)$ and air flow rate $\left(\dot{m}_{\mathrm{a}}\right)$ by:

$$
\Phi=\frac{\dot{m}_{\mathrm{f}}}{\dot{m}_{\mathrm{a}}} \cdot A F R_{\mathrm{st}} .
$$

The "minimum" function (min) in Eq.(8) is used to consider the fact that at non-stoichiometric conditions, there will be unburnt fuel or air depending on $\Phi$. In the GTMC, fuel is injected through a high pressure line with a large pressure drop, therefore the pressure oscillations in the combustion chamber have little effect on the fuel feed lines. 
Hence the fuel flow rate can be regarded as being constant. The variables in Eq.(8) are decomposed into mean $(\bar{x})$ and fluctuation $\left(x^{\prime}\right)$ by $x=\bar{x}+x^{\prime}$, with $\overline{x^{\prime}}=0$, then from Eq.(8) the heat release fluctuation becomes:

$$
\dot{Q}^{\prime}=\frac{\Delta h_{\mathrm{g}}^{\circ}}{A F R_{\mathrm{st}}} \cdot\left[(\min (\Phi, 1)-\overline{\min (\Phi, 1)}) \cdot \overline{\dot{m}_{\mathrm{a}}}+\left(\min (\Phi, 1) \cdot \dot{m}_{\mathrm{a}}^{\prime}-\overline{\min (\Phi, 1) \cdot \dot{m}_{\mathrm{a}}^{\prime}}\right)\right] .
$$

Depending on the value of equivalence ratio, specifically the value of $\min (\Phi, 1)$, it can be shown that Eq.(10) can simplified to:

$$
\dot{Q}^{\prime}= \begin{cases}0 & \text { if } \max (\Phi)<1 \\ \frac{\Delta h_{\mathrm{g}}^{\circ}}{A F R_{\mathrm{st}}} \cdot \dot{m}_{\mathrm{a}}^{\prime} & \text { if } \min (\Phi)>1\end{cases}
$$

The interpretation of Eq.(11) is illustrated in figure 8. Since the fuel supply rate is assumed to be constant, when air supply is abundant, the resulting heat release rate is constrained by the amount of fuel and hence is kept constant. When the air flow rate is lower than that of the stoichiometric air flow rate, the amount of fuel that is consumed will depend on the air flow rate instead, and hence the heat release rate will be proportional to the air flow rate. In a near stoichiometric condition, the fluctuation of air flow rate above and below stoichiometric value will result in an alternation between steady and unsteady heat release rates.

In the cases where there are non-zero heat release rate fluctuations in a rich mixture, Eq. (11) indicates that $\dot{Q}^{\prime}$ is directly proportional to air mass flow rates. In the present model, the fluctuation of mass flow rate can be related to the fluctuation of swirler exit velocity $\left(U_{1}^{\prime}\right)$ with a convection time delay $\left(\tau_{\mathrm{c}}\right)$ :

$$
\dot{m}_{\mathrm{a}}^{\prime}=U_{1}^{\prime}\left(t-\tau_{\mathrm{c}}\right) \cdot \rho_{0} \cdot A_{1}
$$

where $\tau_{\mathrm{c}}$ represents the time for fluid to travel from injector exit to flame position.

The complex flow pattern across the swirler vanes in the injector are neglected. Following the same procedure as shown in the derivation of Eqs.(C.6) and (C.7), the heat release model becomes:

$$
\frac{d \dot{Q}^{\prime}}{d t}=\frac{\Delta h_{\mathrm{g}}^{\circ}}{A F R_{\mathrm{st}}} \frac{d \dot{m}_{\mathrm{a}}^{\prime}}{d t}=\left.\frac{\Delta h_{\mathrm{g}}^{\circ}}{A F R_{\mathrm{st}}} \frac{A_{1}}{l_{1}} \cdot\left(P_{0}^{\prime}-P_{2}^{\prime}\right)\right|_{t-\tau_{\mathrm{c}}} .
$$

\subsection{Determination of convection time delay $\tau_{\mathrm{c}}$}

In the current model, the convection time delay $\tau_{\mathrm{c}}$ is estimated to be the ratio of mean lift-off height $\bar{H}_{\text {lo }}$ and mean injector exit velocity $\bar{U}_{1}$ :

$$
\tau_{\mathrm{c}}=\frac{\bar{H}_{\mathrm{lo}}}{\bar{U}_{1}} .
$$

The mean lift-off height is estimated by the assumptions that: $i$ ) the axial velocity magnitude decreases along the height of the combustion chamber, the rate of decrease is that of an axial jet $\left(\sim x^{-1}\right)$ [42] in far field; ii) flame is stabilized at the location where the local mean velocity equals to the turbulent burning velocity of the fuel $s_{\mathrm{T}}$; $\left.i i i\right)$ the ratio between turbulent $\left(s_{\mathrm{T}}\right)$ and laminar $\left(s_{\mathrm{L}}\right)$ burning velocity is constant despite the local perturbations. With these assumptions, $\bar{H}_{\text {lo }}$ can be calculated as:

$$
\bar{H}_{\mathrm{lo}}=H_{\mathrm{ref}} \cdot\left(\frac{\bar{U}_{1}}{s_{\mathrm{T}}}\right)
$$

where $H_{\text {ref }}$ is a reference length scale. We can then rewrite Eq.14 into:

$$
\tau_{\mathrm{c}}=H_{\mathrm{ref}} \cdot\left(\frac{\bar{U}_{1}}{s_{\mathrm{T}}}\right) \cdot \frac{1}{\bar{U}_{1}}=\frac{H_{\mathrm{ref}}}{\left(s_{\mathrm{T}} / s_{\mathrm{L}}\right) s_{\mathrm{L}}},
$$


which means that the convection time delay is independent of mean flow velocity. Instead, $\tau_{\mathrm{c}}$ is only a function of laminar burning velocity $s_{\mathrm{L}}$ and the ratio of turbulent to laminar burning velocity $s_{\mathrm{T}} / s_{\mathrm{L}}$.

\subsection{Summary of the governing equations}

In the previous sections, we have individually derived the pressure equations of the plenum and combustion chamber as well as the heat release term. They can be coupled by substituting Eq. (13) into Eq. (6), yielding the governing equations of pressure in the system:

$$
\begin{gathered}
\frac{d^{2} P_{0}^{\prime}}{d t^{2}}+\omega_{0}^{2} \cdot P_{0}^{\prime}=\omega_{0}^{2} \cdot P_{2}^{\prime} \\
\frac{d^{2} P_{2}^{\prime}}{d t^{2}}+2 \zeta_{2} \cdot \omega_{2} \cdot \frac{d P_{2}^{\prime}}{d t}+\omega_{2}^{2} \cdot P_{2}^{\prime}=\left.\mathscr{L} \cdot \Theta \cdot\left(P_{0}^{\prime}-P_{2}^{\prime}\right)\right|_{t-\tau_{\mathrm{c}}}
\end{gathered}
$$

with $\mathscr{L}$ representing all burner-specific parameters:

$$
\mathscr{L}=\frac{\gamma_{2}-1}{V_{2}} \cdot \frac{A_{1}}{l_{1}}
$$

and $\Theta$ representing all parameters that are fuel specific:

$$
\Theta=\frac{\Delta h_{\mathrm{g}}^{\circ}}{A F R_{\mathrm{st}}} .
$$

Consider the case when pressure oscillations in the plenum and the combustion chamber are sinusoidal, then they can be written in a complex form:

$$
P_{0}^{\prime}=\left|P_{0}^{\prime}\right| \cdot \exp (\jmath \omega t), \quad P_{2}^{\prime}=\left|P_{2}^{\prime}\right| \cdot \exp \left(\jmath\left(\omega t-\psi_{20}\right)\right)
$$

where $\psi_{20}$ is the phase angle between $P_{0}^{\prime}$ and $P_{2}^{\prime}$. Substitution of Eq. (18) into Eq. (17) leads to:

$$
\begin{gathered}
-\omega^{2}+\omega_{0}^{2}=\omega_{0}^{2} \cdot \mathscr{P}, \\
-\omega^{2}+2 \zeta_{2} \cdot \omega_{2} \cdot \jmath \omega+\omega_{2}^{2}=\mathscr{L} \cdot \Theta \cdot \exp \left(-\jmath \omega_{\mathrm{r}} \tau_{\mathrm{c}}\right) \cdot(1 / \mathscr{P}-1),
\end{gathered}
$$

where $\mathscr{P}$ is the complex ratio of the two pressure fluctuations that is defined as:

$$
\mathscr{P}=\frac{P_{2}^{\prime}}{P_{0}^{\prime}}=\frac{\left|P_{2}^{\prime}\right|}{\left|P_{0}^{\prime}\right|} \cdot \exp \left(\jmath \psi_{20}\right) .
$$

In Eqs. (19a) and (19b) the only unknowns are the system instability frequency $\omega$ and complex pressure ratio $\mathscr{P}$. All other parameters can be estimated from the configuration and operating conditions. Hence mathematical closure is achieved. If we further eliminate $\mathscr{P}$ by substituting Eq.(19a) into Eq.(19b), the governing equation for the combustion instability frequency $\omega$ alone will be:

$$
-\omega^{2}+2 \zeta_{2} \cdot \omega_{2} \cdot \jmath \omega+\omega_{2}^{2}=\mathscr{L} \cdot \Theta \cdot \exp \left(-\jmath \omega_{\mathrm{r}} \tau_{\mathrm{c}}\right) \cdot\left(\frac{\omega^{2}}{\omega_{0}^{2}-\omega^{2}}\right) .
$$

\section{Model Predictions and Comparisons with Experimental Observations}

In the following section, the proposed model is used to predict the trends in the changes of combustion instability frequency when certain parameters of the system are changed. Results are compared with the experimental data. 


\subsection{Model parameters for the GTMC}

The first step towards the application of the reduced order model on the GTMC is the estimation of model parameters. To solve the instability frequency equation, Eq.(21), the values of several constants are needed. Geometric parameters based on the dimensions of the GTMC are provided in table 4 along with some of the thermodynamic parameters.

Table 4: Values of the parameters of the proposed reduced order model in GTMC operating condition

\begin{tabular}{ccccc}
\hline Component & Plenum & Injector & Chamber & Exhaust \\
Subscript & 0 & 1 & 2 & 3 \\
\hline \hline Length $(l)[\mathrm{cm}]$ & 6.5 & 3.6 & 11.0 & 5.0 \\
Diameter $(D)[\mathrm{cm}]$ & 7.90 & $2.37^{*}$ & $8.50^{\dagger}$ & 4.00 \\
Cross-sectional area $(A)\left[\mathrm{cm}^{2}\right]$ & 49.0 & 4.4 & 72.3 & 12.6 \\
Volume $(V)\left[\mathrm{cm}^{3}\right]$ & 319 & 16 & 795 & 63 \\
Temperature $(T)[\mathrm{K}]$ & 294 & 294 & 2000 & 2000 \\
Ratio of heat capacities $(\gamma)$ & 1.4 & 1.4 & 1.3 & 1.3 \\
Speed of sound $(C)[\mathrm{m} / \mathrm{s}]$ & 344 & 344 & 864 & 864 \\
\hline
\end{tabular}

${ }^{*} D_{1}$ is calculated as the equivalent diameter combining both the inner and outer swirler.

+ The combustion chamber has a square cross section and $D_{2}$ is the length of its edge.

The lower heating value of DME is estimated to be $\Delta h_{\mathrm{g}}^{\circ}=27.6 \mathrm{MJ} / \mathrm{kg}$ [43]. The reference length $H_{\text {ref }}$ is taken as the estimated lift-off height of $5 \mathrm{~mm}$. Turbulent burning velocity $s_{\mathrm{T}}$ is estimated to be $5 \mathrm{~m} / \mathrm{s}$, which is about 10 times larger than the corresponding laminar burning velocity of DME at $\Phi=1.2$. This results in a convection time scale $\tau_{\mathrm{c}} \approx 1 \mathrm{~ms}$. In addition, the Helmholtz resonance frequencies of plenum and combustion chamber are calculated using Eqs.(2) and (5):

$$
\begin{gathered}
\omega_{0}=\sqrt{\frac{C_{0}^{2} A_{1}}{V_{0} l_{1}}}=2129 \mathrm{rad} / \mathrm{s} \approx 339 \mathrm{~Hz}, \\
\omega_{2}=\sqrt{\frac{\gamma_{2}-1}{\gamma_{0}-1}\left(\frac{C_{0}^{2} A_{1}}{V_{2} l_{1}}\right)+\left(\frac{C_{2}^{2} A_{3}}{V_{2} l_{3}}\right)} \approx 796 \mathrm{~Hz} .
\end{gathered}
$$

It should be noticed here that both $\omega_{0}$ and $\omega_{2}$ correspond to certain observed features in figure 3 . For example, a mode in the range of $300 \sim 350 \mathrm{~Hz}$ was observed in plenum. Similarly, some feature near $800 \mathrm{~Hz}$ was observed in combustion chamber. However, not all spectral peaks in figure 3 could be explained by the Helmholtz theory.

The calculation of damping coefficient involves estimating the heat transfer coefficients. With an estimated mean axial velocity in the combustion chamber $\left(U_{2}\right)$ of $10 \mathrm{~m} / \mathrm{s}$ and glass wall temperature of $500 \mathrm{~K}$, the non-radiation thermal resistance $\Omega_{\mathrm{i}}$ defined in Eq.(D.1) is $2.81 \mathrm{~K} / \mathrm{W}$. With an estimated emissivity of 0.5 , the radiation thermal resistance $\Omega_{\mathrm{ii}}$ is determined to be $0.1 \mathrm{~K} / \mathrm{W}$. This calculation indicates that the major path of heat transfer in the current setup is heat radiation, as the radiation thermal resistance is much smaller than the non-radiation thermal resistance. The total thermal resistance $\Omega$ is $0.1 \mathrm{~K} / \mathrm{W}$ and the estimated damping coefficient $\zeta_{2}$ from Eq. $(7)$ is then 0.016 . 


\subsection{Reference at case $R 1$}

To predict the trend of changes in combustion instability, a reference case needs to be established. Here the case R1 is chosen, because of its distinctive unstable behavior. Equation (19) is solved with the estimated parameters. The solution contains two variables: the complex instability frequency $\omega$ and complex pressure ratio $\mathscr{P}$. The real part of $\omega, \omega_{\mathrm{r}}$, is the instability frequency observable by experiments. The magnitude of $\mathscr{P},|\mathscr{P}|$, is the ratio of the pressure amplitude in plenum and combustion chamber. The complex phase angle of $\mathscr{P}, \psi_{20}$, is the phase angle between the pressure in plenum and combustion chamber. These predicted variables are compared with experimental data in Chen and Driscoll [36] and the results are shown in table 5.

Table 5: Comparison of instability frequency between predictions of proposed model and experimental data for case R1.

\begin{tabular}{cccc}
\hline & Model prediction & Experiment $^{*}$ & Difference \\
\hline \hline$\omega_{\mathrm{r}}$ & $329 \mathrm{~Hz}$ & $330 \mathrm{~Hz}$ & $<0.3 \%$ \\
$|\mathscr{P}|$ & 0.63 & 0.5 & $<26 \%$ \\
$\psi_{20}$ & $76^{\circ}$ & $50^{\circ}$ & $<52 \%$ \\
\hline \multicolumn{4}{r}{ " Data from Chen and Driscoll [36]. }
\end{tabular}

From table 5 we can see that the proposed model accurately predicted the measured instability frequency. The predictions of complex pressure ratio $\mathscr{P}$ have larger deviations from experiment data. Specifically, the magnitude of the pressure ratio is $40 \%$ smaller and the phase angle is $50 \%$ larger than the experiment. Such discrepancies can be caused by a variety of reasons. For example, the complex geometry in the dual-swirler is neglected, which may have effects over the interactions between the pressure in plenum and combustion chamber. Even considering the differences, we can see that the proposed model is able to provide predictions that are in the same range as the experimental observations.

\subsection{Effect of varying the dimensions of the GTMC}

With all the parameters estimated at the reference point (case R1, DME flame, $\Phi=1.2, \dot{m}_{\mathrm{a}}=282 \mathrm{~g} / \mathrm{min}$ ), we explore the effect of changing geometric parameters of the GTMC in our model and compare the results with the experimental data provided by Allison et al. [26].

The first parameter of interest is the plenum volume $V_{0}$. In figure 9 it shows that our prediction (solid line) follows the same trend as the experimental data. Specifically the instability frequency decreases as the volume of plenum increases. This is expected because from Helmholtz analysis we know that as the plenum volume increases, the characteristic frequency will decrease. Our combustion instability frequency $(\omega)$ is closely related to the Helmholtz characteristic frequency of the plenum $\left(\omega_{0}\right)$ by Eq. 21 , hence it will also decrease as $\omega_{0}$ decreases.

The results in figure 9 are shown on a normalized base. Due to the differences in fuel type and wall configuration between the current study and the cited previous study, the instability frequencies of the system at standard GTMC dimension can be very different. To better compare the trend of the two data sets, the normalization procedure was carried out. The reference frequency $\omega_{\text {ref }}$ is taken from the system instability frequency when all GTMC dimensions are at their normal values, as shown in table 4. For example, the experimental data in figure 9 was normalized by 
$322 \mathrm{~Hz}$ and the model prediction was normalized by $311 \mathrm{~Hz}$. In this way, the experimental data and model prediction would always coincide at the point $(1,1)$ in the normalized space. The same methodology has been employed for all subsequent comparisons.

The difference between our model prediction and experimental data lies at the slope of the curve. Our model as well as Helmholtz theory predicts that frequency decreases at a rate of $V_{0}^{-0.5}$ whereas in the experimental data the frequency decreases at a rate of $V_{0}^{-0.25}$. Sensitivity analysis has been conducted and we found that varying $\zeta_{2}$ would not mitigate this discrepancy. We have also varied the value of $\tau_{c}$ from $1 \mathrm{~ms}$ to $2 \mathrm{~ms}$. Even though the magnitude of the predicted frequency gets closer to the corresponding experimental value, the difference of slope is still evident. The exact reason of this discrepancy has yet been identified.

Next we examine the effect of changing the dimensions of the exhaust duct on the instability frequency. In figure 10 the predicted results are compared against Allison's data [26] on a normalized basis.

If the combustion instability frequency is purely attributed to the influence of the plenum, the change in exhaust duct would have no effect on the instability. However, experimental data show that the geometry of the exhaust duct is an important factor. Because our model considers the interaction between plenum and combustion chamber, the effect of the exhaust duct is captured. Figure 10 shows that our model predictions captured the measured trends when exhaust duct diameter and length are changed.

It was reported by Allison et al. [26] that the change of combustion chamber length from $30 \%$ to $300 \%$ of its original length results in less than $5 \%$ of change in measured combustion instability frequency. The predictions of the reduced order model in the same range of combustion chamber length is shown in figure 11.

The solid line in figure 11 shows that the instability frequency decreases as the combustion chamber length is increased. However, if $l_{2}$ is within $\pm 50 \%$ of its reference value, the instability frequency remains within $\pm 5 \%$ of the original instability frequency. Even though the change in our model prediction (solid line) is larger than the measured value (dash line), the model correctly predicts that the system instability frequency is insensitive to the change of combustion chamber volume.

In this first part of this section, it is shown that the instability frequency is sensitive to plenum volume and exhaust duct dimensions. This is because these parameters directly affect the Helmholtz frequency of plenum $\left(\omega_{0}\right)$ and combustion chamber $\left(\omega_{2}\right)$, which are the important coefficients in Eq.(19). Changes in combustion chamber length and hence its volume will also affect $\omega_{2}$. However because $V_{2}$ is also present on the right hand side of Eq.(19b), the effect of changing $\omega_{2}$ is partially offset. This results in the relative insensitivity of $\omega$ on $l_{2}$ and $V_{2}$.

\subsection{Effect of varying equivalence ratio}

Section 2.3 summarized our experimental observations of the change in system dynamics as the equivalence ratio is changed. Similar to the observations made by Allison et al. [27], the fuel-lean flame L1 with $\Phi=0.75$ was considered to be "stable" because of its very small pressure oscillation. The flame S1 with $\Phi=1.0$ is "intermittent" because of its intermittent pressure fluctuations, while the flame R1 and R2 are fuel rich $(\Phi=1.2)$ and are "unstable" because of their very large pressure fluctuations.

In the proposed model, the influence of equivalence ratio on combustion instability is through its influence on heat release rate. The heat release model is summarized by Eq.(11). This equation states that when the mixture 
is very lean $(\max (\Phi)<1)$, the heat release rate is constant, and its fluctuation is zero. Considering that the heat release rate fluctuation is the driving force for combustion chamber pressure fluctuation, $\dot{Q}^{\prime}=0$ means that Eq. (4) will have a trivial solution of $P_{2}^{\prime}=0$. This in reality means that there will not be combustion instability present. When the mixture is rich in fuel, heat release rate will fluctuate if the air flow rate oscillates, thus providing the possibility of combustion instability if Rayleigh criterion is also satisfied. Lastly, if the mixture is near stoichiometric condition and the air flow rate is unsteady, combustion instability can only occur in the instances when the mixture at flame is fuel rich.

In conclusion, the proposed model provides a possible explanation for the experimental observation that equivalence ratio is closely related to the occurrence of instability in the GTMC. At the same time, it should be understood that such prediction is based on many assumptions as detailed in section 3.4. Some of these assumptions may be a simplified view of the complex interactions present in the GTMC, our proposed model nonetheless provides a theoretical base to understand fundamental physical processes in this burner.

\subsection{Effect of varying combustion chamber wall configuration}

Experimentally it has been found that the combustion instability frequency is affected by the configuration of the walls of the combustion chamber. For example, in the study by Allison et al. [27], the pressure measurement was done with three steel walls and one glass wall, and for case R1 the frequency was about $360 \mathrm{~Hz}$. In the study by Chen and Driscoll [36] the configuration consisted of three glass walls and one steel wall, and the resultant frequency was around $330 \mathrm{~Hz}$. In present study, the combustion chamber has four glass walls and the measured instability frequency is about $320 \mathrm{~Hz}$.

In the proposed model, wall heat loss affects the instability frequency through its influences on the combustion chamber damping ratio $\zeta_{2}$. Specifically, different wall configurations alter the total thermal resistance $\Omega$, and Eq. 7 predicts that the damping ratio $\zeta_{2}$ will decrease with a increasing total thermal resistance of the walls.

In the meantime, it should also be noted that the wall configuration also affects the leakage of products through the walls, which is neglected in the present model. In conclusion, a precise prediction of the relation between wall configuration and damping ratio $\zeta_{2}$ is difficult. Nonetheless, since we acknowledge that there is a correlation between the two variables, we solved the governing equation Eq.(21) through a range of $\zeta_{2}$ values and plotted the solution of instability frequency $\omega$ in figure 12 . The values of experimentally measured instability frequency for different wall configurations are also plotted onto the same figure.

The proposed model predicts that the instability frequency increases with an increase in the combustion chamber damping coefficient.

\subsection{Effect of varying convection time delay}

In the current model, the convection time delay $\tau_{\mathrm{c}}$ is a very important variable. For example, the only explicit dependence of the combustion instability frequency on laminar burning velocity is through convection time delay, as shown in equation 16. However, it is very difficult to directly measure the convection time delay. The exact determination of this variable in experiment would require the tracking of fluid parcels in the turbulent flame. This is not achievable partially due to the recess of the injector exit below the burner surface, which makes certain portion of the flow field optically inaccessible. One way of estimating the convection time delay would be the time lag between 
plenum pressure oscillation and total flame surface area oscillation, because it roughly shows the time it takes for a disturbance in plenum to affect the flame. A sample of this data in temporal domain for case R1 is plotted in figure 13 .

From the data we observed that the averaged time delay between the plenum pressure and total flame surface area is in the range around $1 \mathrm{~ms}$, which is in accordance with our estimated value. However, due to spectral noises, the time lag between these two signals could not be accurately determined in the temporal domain without filtering. Also this time lag is only a rough approximation to the convection time delay. Thus the sensitivity of our model to the convection time delay must be investigated. Figure 14 shows the predicted relationship between instability frequency $\omega$ and convection time delay $\tau_{\mathrm{c}}$. One period of the pattern is presented. In this figure the dependence of $\omega$ on $\tau_{\mathrm{c}}$ is not monotonic. Hence the dependence of $\omega$ on $s_{\mathrm{L}}$ is determined by the specific operating condition. Furthermore, the system of equations does not have a converged solution when $\tau_{c} \omega_{\text {ref }}$ approaches $\pi$.

The relationship between instability frequency and convectional time delay in a different experiment was shown in the work of Durox et al. [44]. There, instead of a "U" shaped curve as what we predicted, the $\omega-\tau$ curve was monotonically decreasing in each period. The difference in the curve shape is likely due to the different acoustic boundary conditions that the two experiments have. However, the two curves do share some features such as the critical points at the boundary of periodicity.

\subsection{Effect of varying mass flow rate}

There is no explicit dependence on air mass flow rate in our governing equation Eq.(21). However, the air mass flow rate, and hence the mean velocity, does play a key role in determining the damping term in the equations. Specifically, as the mass flow rate increases, the Reynolds number in the combustion chamber increase accordingly. This will result in a increase in the convection heat transfer coefficient and an increased damping coefficient.

If we consider the case where the combustion chamber has four steel walls, then heat convection will be the dominant heat transfer mechanism. In this case, the dependence of instability frequency on mean flow rate is plotted in figure 15 as predicted by the proposed model.

Figure 15 indicates that the predicted instability frequency increases monotonically with the increase of air flow rate. This trend agrees with the experimental observations made by Allison et al. [27] for different fuels. However, the magnitude of the change in instability frequency predicted by the proposed model is much smaller than that of the experimental data. This means that at this stage, the sensitivity of the proposed model to mass flow rate variation is still insufficient. Currently the viscous effects of the mean flow has been neglected, which may be a contributing factor to this discrepancy. This opens an area for future improvement.

\section{Summary and Conclusions}

The DLR gas turbine model combustor (GTMC) was operated with DME fuel at four operating conditions: rich (R1), lean (L1), stoichiometric (S1), and reduced flow rate (R2). Highspeed PIV-PLIF-pressure measurements were carried out at a sustained frequency of $4 \mathrm{kHz}$. The spectra of pressure, heat release, and velocity vectors were investigated. A reduced order model that is based on multi-chamber Helmholtz analysis was proposed. Unlike previous models that consider only one chamber or require empirical calibration, the proposed reduced order model 
utilizes a set of coupled ODEs that consider a system with two chambers (plenum and combustion chamber) and two constrictions (injector and exhaust duct). Supplied with the realistic values of GTMC parameters, the model was used to predict the instability frequency and complex pressure ratio at a fixed operating condition (case R1). It was also used to explore the effect of geometry, equivalence ratio, and mass flow rate on combustion instability.

We conclude that:

- The PLIF data showed that the spectrum of flame surface density oscillations has a sharp spike at the same frequency as the spike in the pressure spectrum. This indicates that heat release rate and pressure fluctuations are related. The kilohertz PIV data showed that the velocity field also has a peak at the instability frequency in unstable flames.

- Velocity spectrum analysis revealed that combustion instability changes the axial and radial velocity distribution. In an unstable flame the major dynamics happened in the radial direction, with velocity undergoing a large oscillation radially. In a stable flame the velocity oscillation was more evenly distributed between axial and radial directions.

- PIV data showed that there was a strong PVC in the stable flame L1 and a weak PVC in the unstable flame R2. The existence of combustion instability was shown to be independent of the presence of PVC.

- The proposed reduced order model provides an explanation for the dependence of the measured frequencies of the combustion instability on several parameters: equivalence ratio, mass flow rate, heat loss through walls, and various geometric dimensions.

- The heat release model hypothesizes that the heat release rate is controlled by the flow rate of the deficient stream. If the fuel flow rate is constant, then in all fuel lean conditions the heat release rate stays constant and combustion instabilities will not occur. This idea is an extension to existing equivalence ratio oscillation theories.

- The model at its current form is tailored to model the GTMC specifically. Additionally, the heat release model is only valid if we ignore the effect of fluid dynamics on the flame and assume one-step chemistry.

\section{Acknowledgments}

We thank Dr. Wolfgang Meier of DLR Stuttgart for permission to use the GTMC design and we thank Prof. Matthias Ihme for helpful collaborations. Support was provided by ONR grant N00014-10-10561 and by DOE-UTSR grant FE0007060. 
(b)

(a)
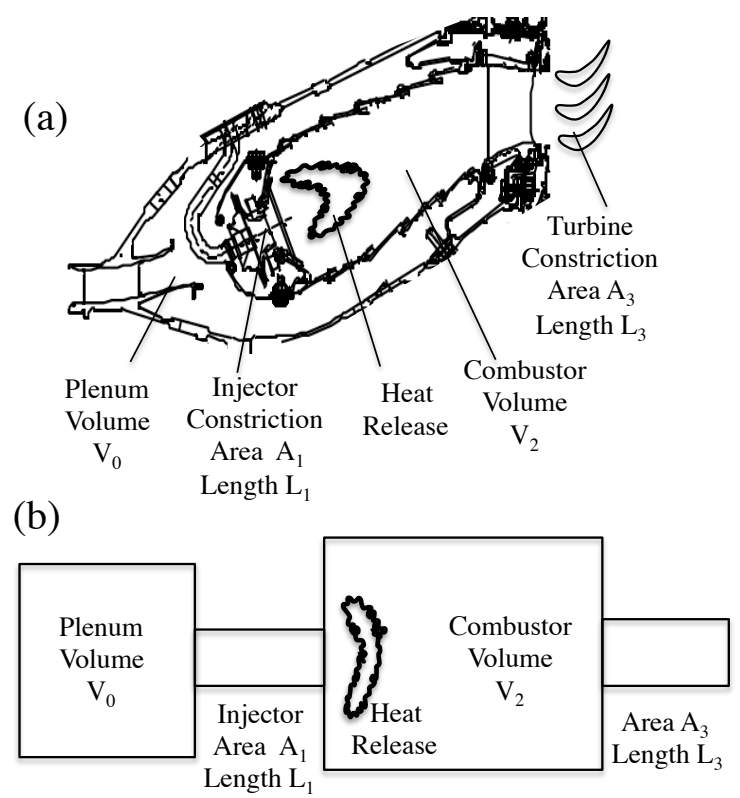

Figure 1: Schematic of the combustor of a gas turbine engine 


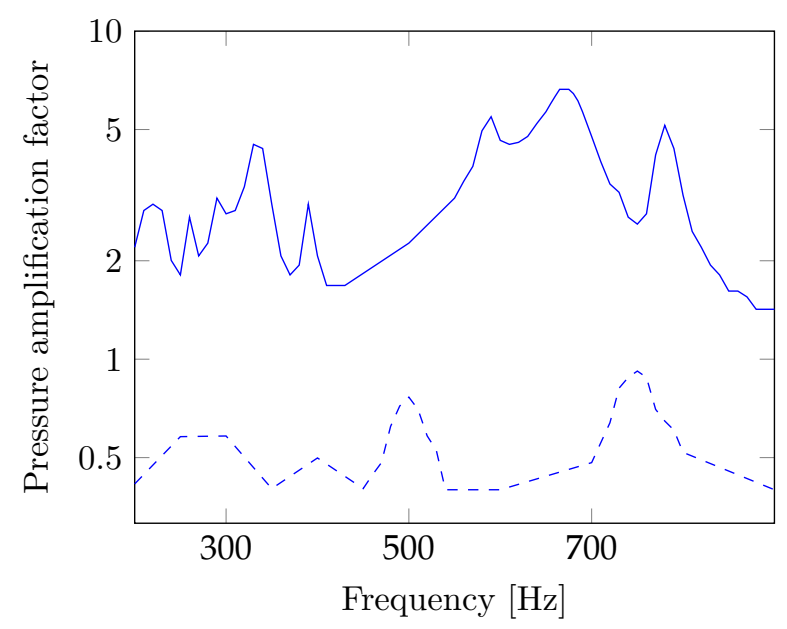

Figure 3: Amplification factor measured in the plenum (solid blue, top curve) and combustion chamber (dashed blue, bottom curve) when a loudspeaker was used to excite each component separately. 


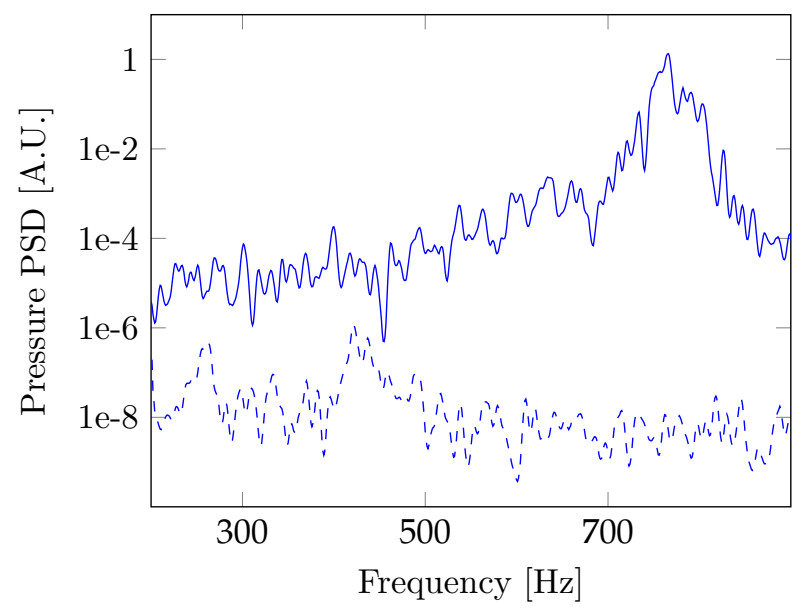

Figure 4: Power spectrum density of plenum and combustion chamber pressure without flame. Data shown here have been smoothed. The solid line denotes the pressure in the plenum and the dashed line denotes the pressure in the combustion chamber. 
7

8

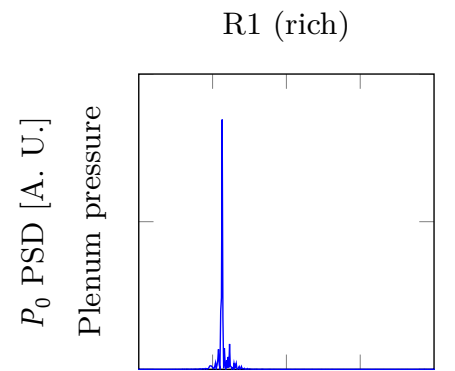

L1 (lean)
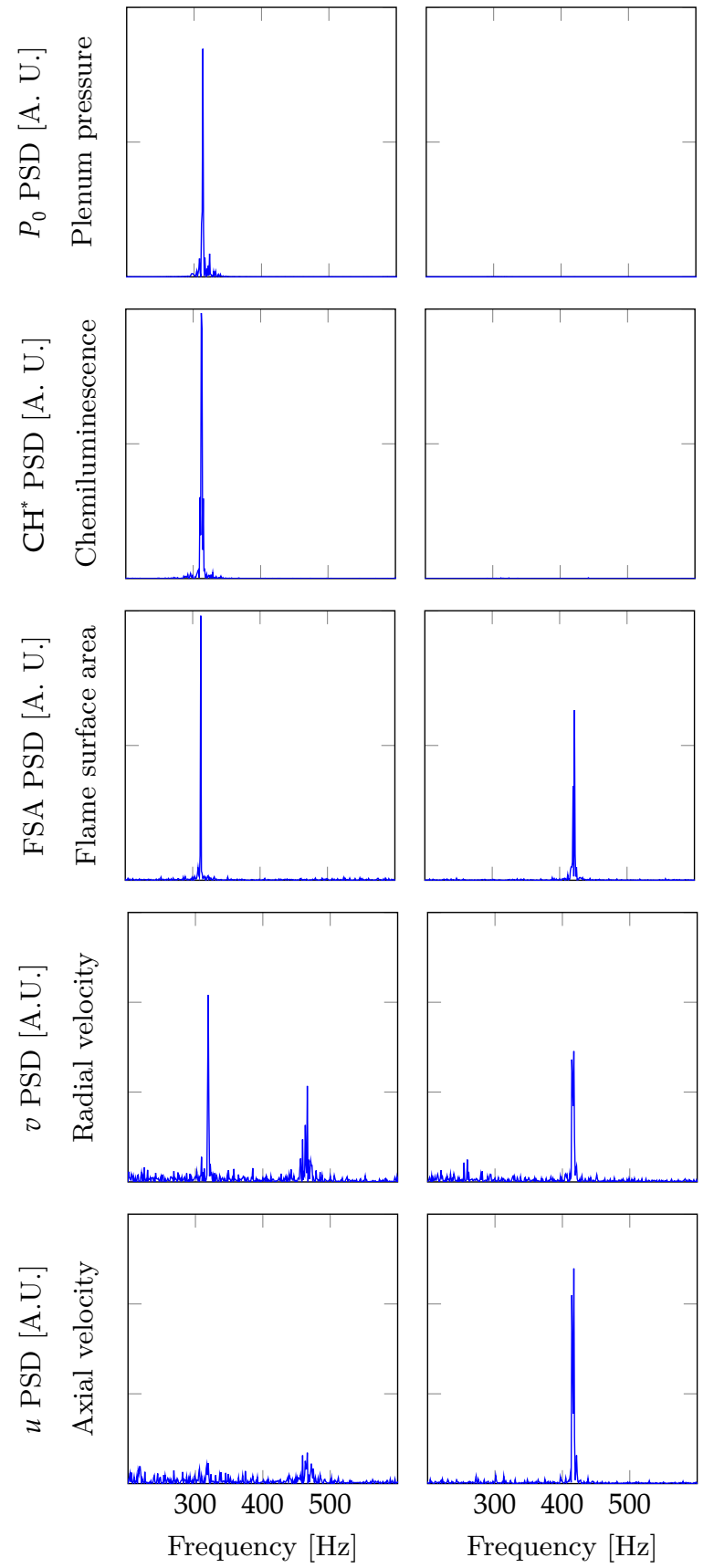

S1 (stoichiometric)

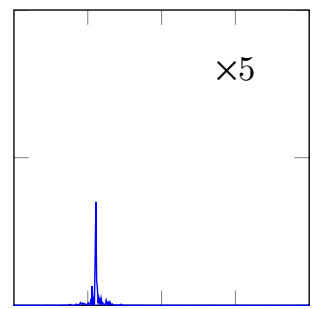

R2 (rich, low flowrate)
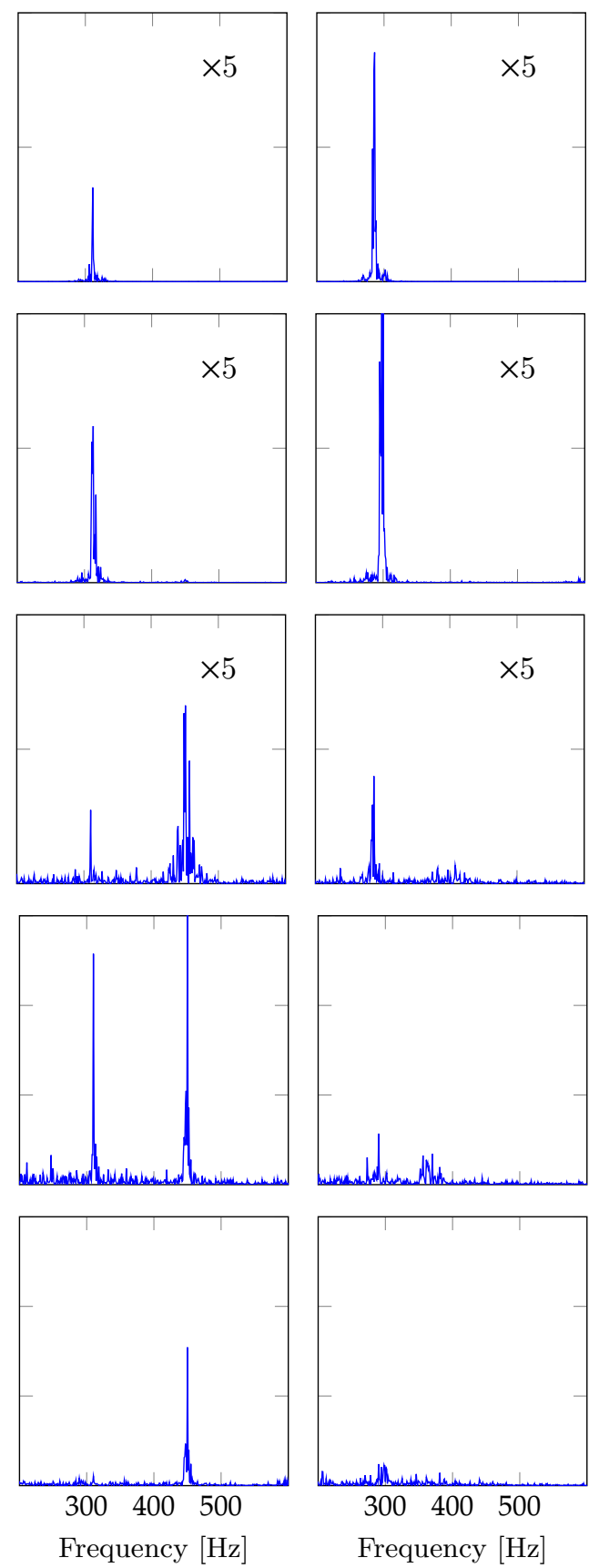

Figure 5: Normalized power spectra for all cases in arbitrary units. First row: plenum pressure, second row: heat release rate $\left(\mathrm{CH}^{*}\right.$ chemiluminescence), third row: flame surface area (FSA) from kilohertz formaldehyde PLIF, fourth row: radial velocity from kilohertz PIV, fifth row: axial velocity. Operating conditions for case R1, L1, S1, and R2 are shown in table 2 . Velocity measurement location is shown in figure 2. 

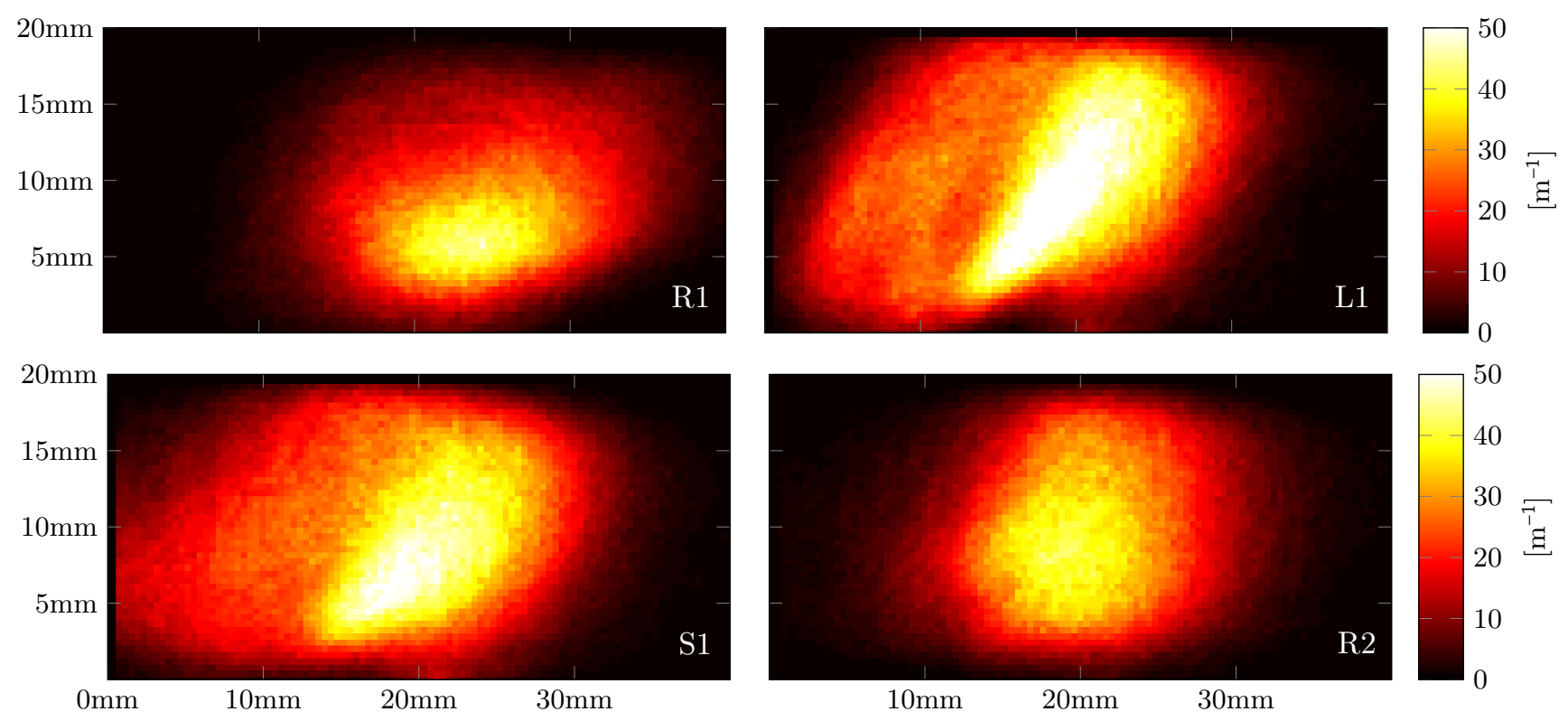

Figure 6: Flame surface density (FSD) for all four cases, in $\left[\mathrm{m}^{-1}\right]$.

Unburnt Mixture Burnt Mixture

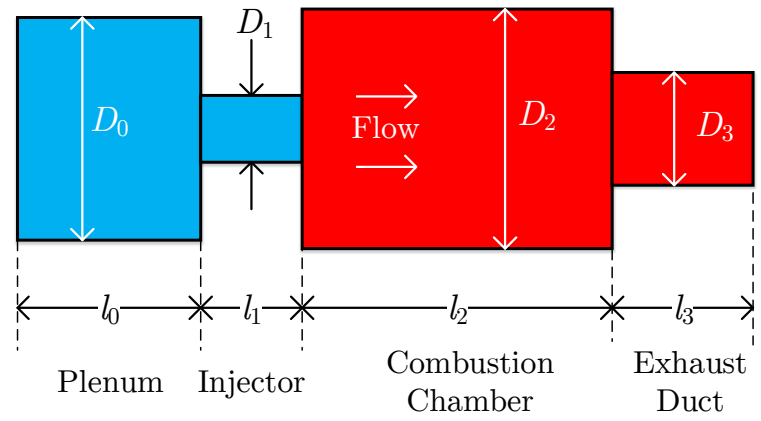

Figure 7: Schematic of the geometry considered in proposed reduced-order model (ROM)

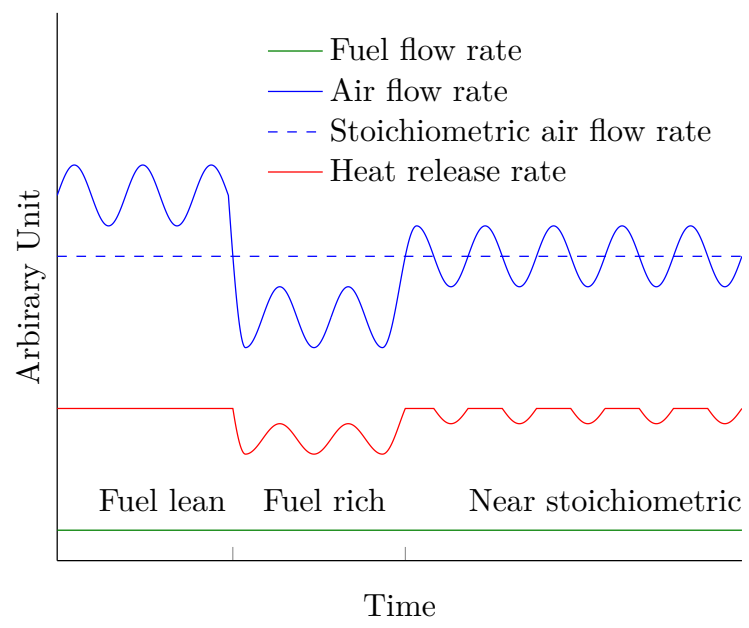

Figure 8: Prediction of Eq.(11) on the fluctuations of heat release rate under different equivalence ratio values 


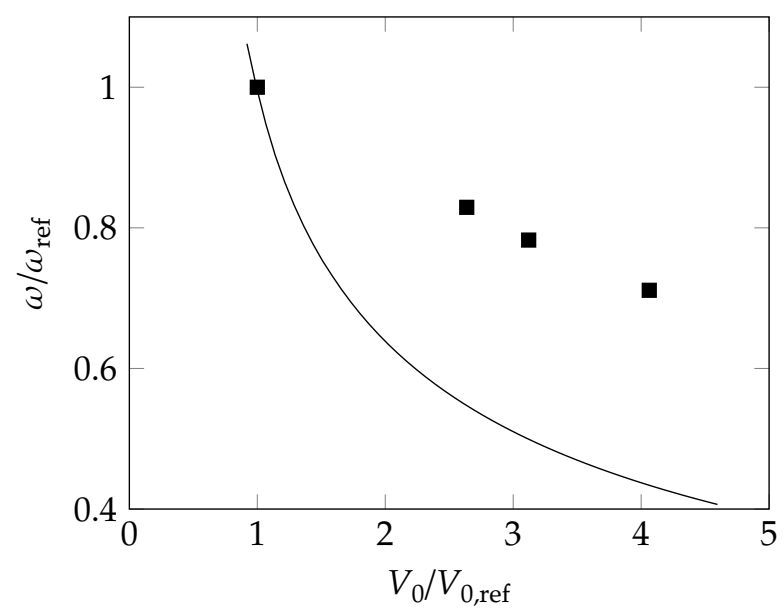

Figure 9: Effect of varying plenum volume $V_{0}$ on the instability frequency: solid squares are experimental data by Allison et al. [26], solid line is prediction made by the proposed model. The choice of reference value $\omega_{\text {ref }}$ for each case is explained in the text in section 4.3 .

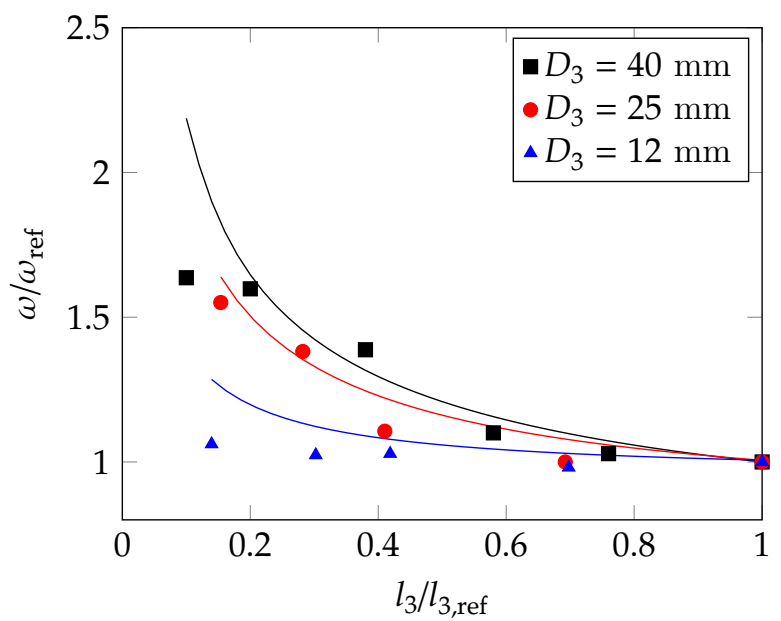

Figure 10: Effect of varying exhaust duct length $l_{3}$ on the instability frequency at different exhaust duct diameter $\left(D_{3}\right)$ : solid symbols are experimental data by Allison et al. [26], solid lines are the prediction made by proposed reduced order model. The choice of reference value $\omega_{\text {ref }}$ for each case is explained in the text in section 4.3 . 


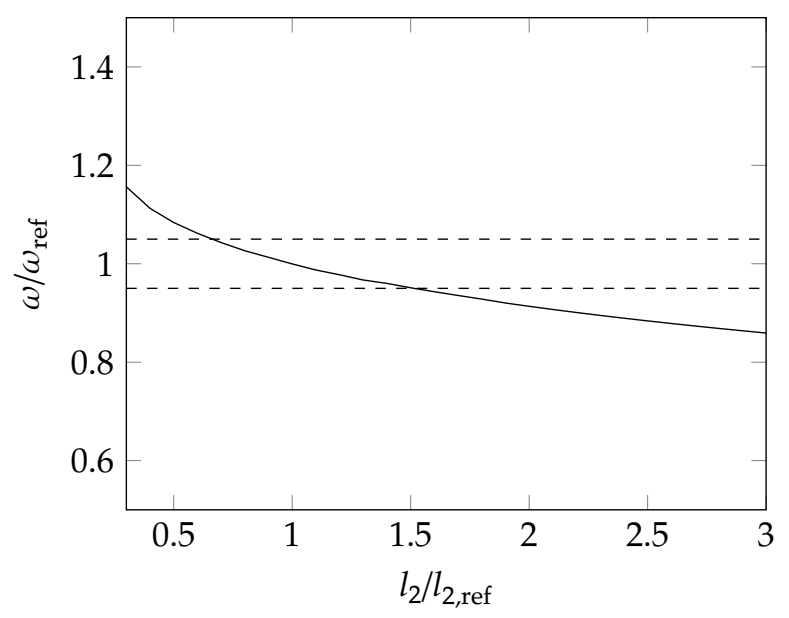

Figure 11: Effect of varying combustion chamber length $l_{2}$ on the instability frequency: solid line is the prediction made by proposed reduced order model, dashed lines are the range of frequencies measured by Allison et al. [26]. The choice of reference value $\omega_{\text {ref }}$ for each case is explained in the text in section 4.3.

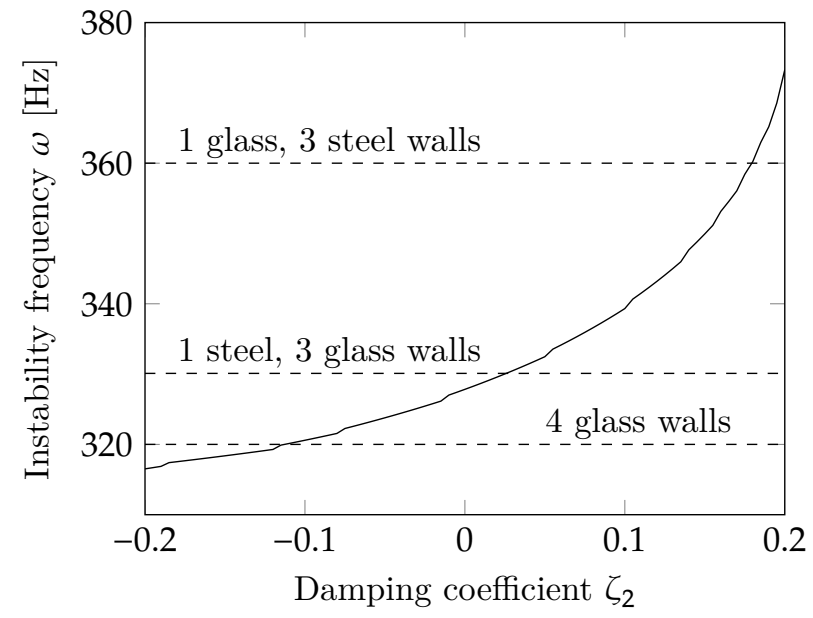

Figure 12: Relationship between predicted instability frequency $\omega$ and combustion chamber damping coefficient $\zeta_{2}$, dashed lines show the measured instability frequency for case R1 with different combustion chamber wall configurations.

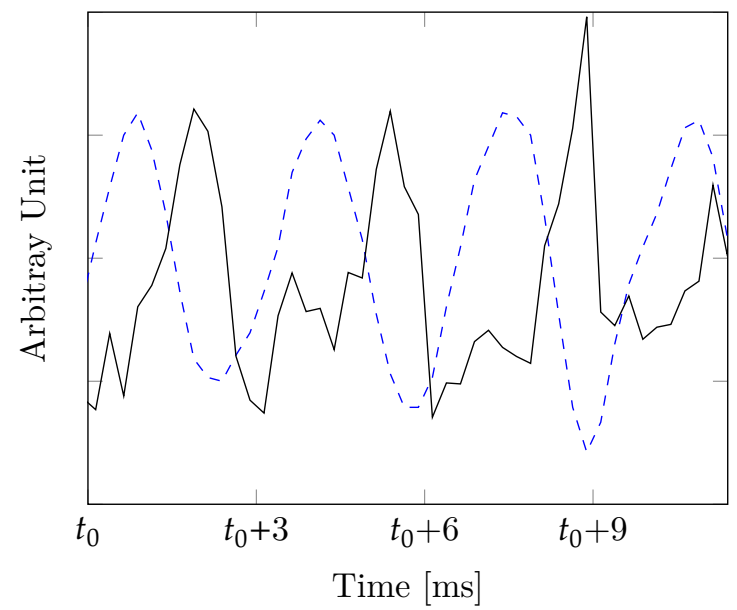

Figure 13: Sample temporal data of plenum pressure (dash line) and total flame surface area (solid line) for case R1. The time step $t_{0}$ was chosen at an arbitrary point. 
50

51

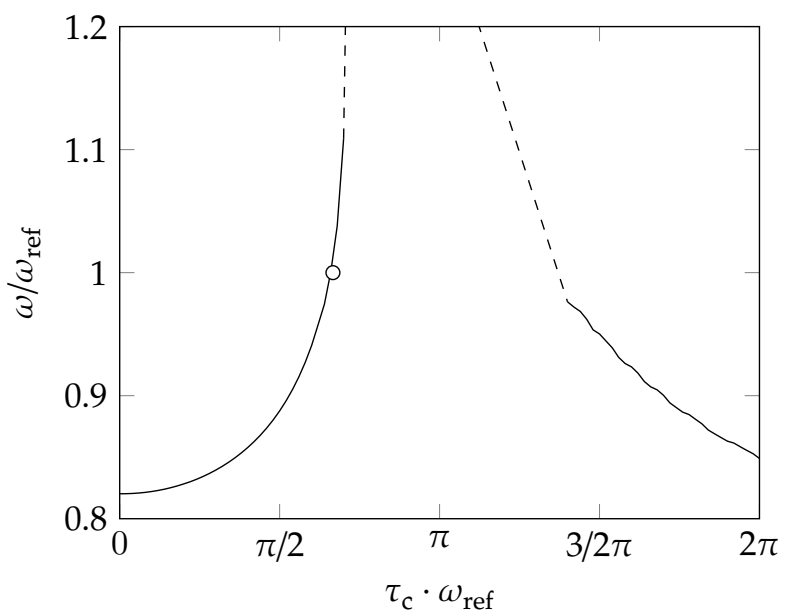

Figure 14: Effect of convection time delay $\tau_{\mathrm{c}}$ on system instability frequency. Black circle indicates the current estimated $\tau_{\mathrm{c}}$ value of 1 ms. Regions where solutions can not converge are indicated by dashed lines. The reference value $\omega_{\text {ref }}$ is $330 \mathrm{~Hz}$.

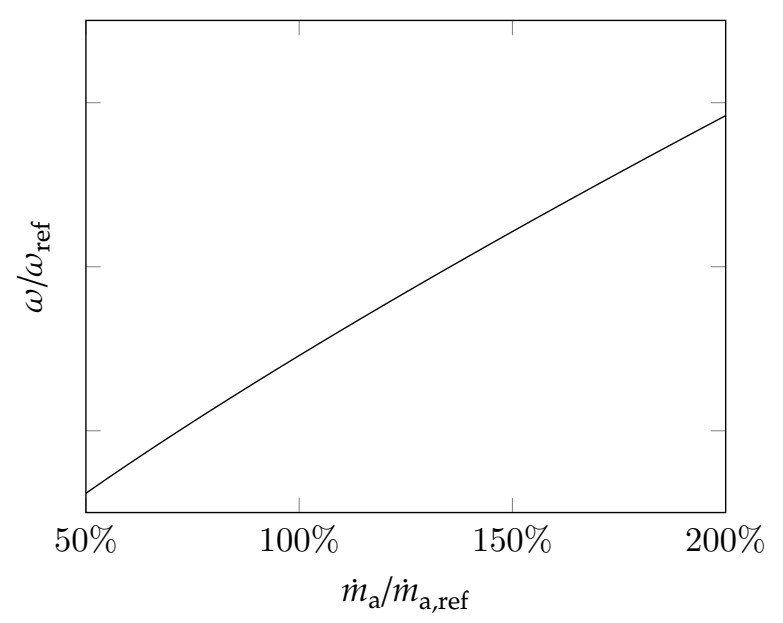

Figure 15: Dependence of predicted instability frequency $\omega$ on air mass flow rate $\dot{m}_{\mathrm{a}}$. Predictions are consistent with measurements [27]. 


\section{A. Details of Optical Diagnostics}

The optical diagnostic system involved in the present study consists of three parts: the PIV, the PLIF, and the $\mathrm{CH}^{*}$ chemiluminescence setups.

The PIV laser beam is produced by a $532 \mathrm{~nm}$ Quantronix Hawk-Duo Nd:YAG laser at a sustained frequency of $8 \mathrm{kHz}$ at $10 \mathrm{~mJ} /$ pulse. The beam is contracted by cylindrical lens (CVI-SCX-50.8-254.3-UV-355-532). A laser sheet with a height of $20 \mathrm{~mm}$ is obtained above the burner surface. The PIV camera (Phantom v9.1) is coupled with a Nikkor 105mm f2.8 macro lens and a narrow band-pass $532 \mathrm{~nm}$ filter (Andover 532FS02-50). It is synchronized with the laser pulse at $8 \mathrm{kHz}$, at which frequency the resolution of the camera is $384 \times 384$ pixels, yielding a scale of $39 \mu \mathrm{m}$ per pixel for a field of view of $15 \mathrm{~mm} \times 15 \mathrm{~mm}$. The resolving power of the camera is determined by a standard 1951 USAF target to be 9 lpmm. With frame-straddling, the the PIV signal is obtained at $4 \mathrm{kHz}$. As shown in figure 2, the PIV field of view is positioned at the exit of the swirler. In this way a fairly uniform flow field was captured in the PIV frame. After processing the PIV images in LaVision Davis software with a $64 \times 64$ pixels window and $50 \%$ overlapping ratio, a matrix of $12 \times 12$ vectors per frame was obtained. All 144 vectors were averaged in axial and radial direction, and the average axial and radial velocity were estimated.

The PLIF laser beam is produced by a $355 \mathrm{~nm}$ Quantronix Hawk-Pro Nd:YAG laser at a sustained frequency of $4 \mathrm{kHz}$ at $10 \mathrm{~mJ} /$ pulse. It was used to excite the formaldehyde molecule that are produced by the decomposition of the DME fuel. The beam passes similar cylindrical lens as the PIV setup, yielding the same beam height of $20 \mathrm{~mm}$ at the burner surface. Before reaching the burner, the $532 \mathrm{~nm}$ PIV beam was merged with the $355 \mathrm{~nm}$ PLIF beam at a dichroic mirror (CVI BSR-35-2025). The PLIF camera (Phantom v711) was coupled with a high speed intensifier (LaVision HS-IRO), both synchronized to the PLIF laser at $4 \mathrm{kHz}$. A Nikkor 105mm f2.8 macro lens with a filter set was installed in front of the intensifier. The filter set consisted of a CG-385 filter that blocks light below $380 \mathrm{~nm}$ and a BG-3 filter that blocks light in the range of $480 \mathrm{~nm}$ to $680 \mathrm{~nm}$. Because the majority of the formaldehyde PLIF signal is in the range of $400 \mathrm{~nm}$ to $500 \mathrm{~nm}$ [45], such a combination of filters for PLIF camera can block the incident laser light at $355 \mathrm{~nm}$ and black body radiation at high wavelengths while passing the PLIF signal. The PLIF image was represented by $508 \times 1016$ pixels (cropped from a $720 \times 1280$ raw image), yielding a scale of $39 \mu \mathrm{m}$ per pixel for a field of view of $20 \mathrm{~mm} \times 40 \mathrm{~mm}$. The resolving power of the combination of camera and intensifier is determined to be $7 \mathrm{lpmm}(70 \mu \mathrm{m}$ resolution).

In a separate measurement, the intensifier was coupled with a Photron SA-Z (type 2100K-M-64GB) camera and triggered at $20 \mathrm{kHz}$. A $430 \mathrm{~nm}$ notch filter (CVI 430-F10-20 T-FYK) was placed in front of the Nikkor 105mm f2.8 macro lens to capture the $\mathrm{CH}^{*}$ chemiluminescence signal emitted by the flame. The field of view of the chemiluminescence measurement was $40 \times 80 \mathrm{~mm}$, and the resolution of the captured image was cropped to $480 \times 960$ pixels, which yields a scale of $83 \mu \mathrm{m}$ per pixel. The resolving power of the chemiluminescence measurement system is 2 lpmm. 


\section{B. Determination of the mode of instability in the combustion chamber of GTMC}

The determination of the model of instability is a important step towards the development of an accurate reduced order model for the combustion instability in the GTMC. It has been previously proposed that the instability mode is of Helmholtz type [20], in this study we were to verify this statement and explore other possible modes as well. The approach we took was two point simultaneous pressure measurements.

For the pressure measurements the operating condition was kept at R1 (air flow rate $282 \mathrm{~g} / \mathrm{min}$, equivalence ratio 1.2). One of the glass walls of the combustion chamber was replaced with a $9.5 \mathrm{~mm}$ thick steel plate. Six pressure taps were drilled into the wall so that pressure transducers could be mounted. Figure B.1 shows the location of these pressure ports in the combustion chamber (point "A" through "F"). The locations of these points on the combustion chamber wall are shown in table B.1. Here the symmetric axis of the burner is taken as $x=0$ and the burner surface is taken as $y=0$. An additional pressure port was drilled into the plenum wall and labeled point "P", also shown in figure B.1. Two PCB piezoelectric pressure transducers were used to make the measurements. Measured signals were passed on to an oscilloscope (LeCroy Waverunner 6100A) for digital recording. The sampling rate of the oscilloscope was set to 25,000 samples per second and 5 seconds of data were recorded during each run. To eliminate hardware errors, one pressure transducer was fixed at point "B" while the other transducer was rotated through all other pressure ports. Further calibration was provided by switching the two pressure transducers while measuring the phase differences between point "B" and "C". Pressure traces were recorded at a fixed burner surface temperature of $322 \mathrm{~K}$.

Table B.1: Pressure measurement point locations* .

\begin{tabular}{lrrrrrr}
\hline Point & A & B & C & D & E & F \\
\hline \hline$x[\mathrm{~mm}]$ & 0 & -19 & 19 & 0 & -19 & 19 \\
$y[\mathrm{~mm}]$ & 19 & 38 & 38 & 57 & 76 & 76 \\
\hline$* x=0$ at burner symmetric axis and $y=0$ at burner \\
$\quad$ surface.
\end{tabular}

Relative phase differences between each measurement point and point "B" were calculated by Fourier transformation and shown in table B.2. It shows that almost all the pressure ports inside the combustion chamber were in phase with each other. And the pressure inside the plenum was lagging by $0.44 \mathrm{~ms}$. Similar behaviors have also been observed by Steinberg et al. [18].

Pressure oscillation amplitudes at each point inside the combustion chamber are plotted in figure B.2.We see that even though the pressure amplitudes decrease slightly as the distance from the burner surface increases, the change is less than $10 \%$.

By far the most widely accepted mode of instability in the GTMC is the Helmholtz type. However standing waves have been observed previously in combustion systems with large aspect ratios [46]. And it was shown that there are inner and outer recirculation zones generated by the injector inside the combustion chamber [15]. These recirculation zones are prone to vortex shedding, which can also generate instabilities. Each of these modes has its own characteristics. If vortex shedding caused instability inside the chamber, then its frequency would be determined 
Table B.2: Relative phase between each measurement point and point B, based on $\omega=330 \mathrm{~Hz}$

\begin{tabular}{cc}
\hline Point & $\begin{array}{c}\text { Phase difference } \\
\text { w.r.t. }\end{array}$ \\
\hline \hline P $\left(\psi_{B}-\psi_{*}\right)$ \\
A & $50^{\circ} \pm 5^{\circ}$ \\
C & $-10^{\circ} \pm 5^{\circ}$ \\
D & $0^{\circ} \pm 5^{\circ}$ \\
E & $0^{\circ} \pm 5^{\circ}$ \\
F & $0^{\circ} \pm 5^{\circ}$ \\
\hline
\end{tabular}

by the Strouhal number:

$$
\text { St }=\frac{f \cdot L}{u},
$$

where $f, L$, and $u$ are characteristic frequency, length, and velocity respectively. If we choose $L$ to be $8.5 \mathrm{~cm}$ (burner surface edge length), $u$ to be $10 \mathrm{~m} / \mathrm{s}$ (average axial velocity [15]), and St to be 0.2 [47], we can estimate the vortex shedding frequency $\left(f_{\mathrm{vtx}}\right)$ to be:

$$
f_{\mathrm{vtx}}=\frac{\mathrm{St} \cdot u}{L} \approx 24 \mathrm{~Hz} .
$$

The result is two orders of magnitude smaller than what we observed in the experiment. Even though this is a very rough estimate, the discrepancy can not be explained by merely the error of estimation. This leads us to the conclusion that the observed instability at $330 \mathrm{~Hz}$ is not caused by vortex shedding.

If a longitudinal standing wave is present in the combustion chamber, we would see the pressure oscillation amplitudes fluctuate from maximum to zero at least once across the combustion chamber height. If the standing wave is a quarter wave, then near the exit duct the pressure oscillation should be close to zero, and the acoustic frequency $f_{\text {std }}$ should be:

$$
f_{\text {std }}=\frac{c}{4 l_{2}} \approx 2 \mathrm{kHz} .
$$

This frequency substantially differs from the measured instability frequency of $330 \mathrm{~Hz}$. The results shown in figure B.2 also contradict the quarter wave theory because pressure amplitudes stay fairly constant across the height of the burner instead of showing a harmonic pattern. Hence the presence of a standing wave in the combustion chamber is also very unlikely.

The small relative phase angles and similar pressure amplitudes inside the chamber are in line with the Helmholtz resonator behaviors. Therefore we reach the conclusion that the dominant instability mode of the combustion chamber is indeed a Helmholtz mode. 


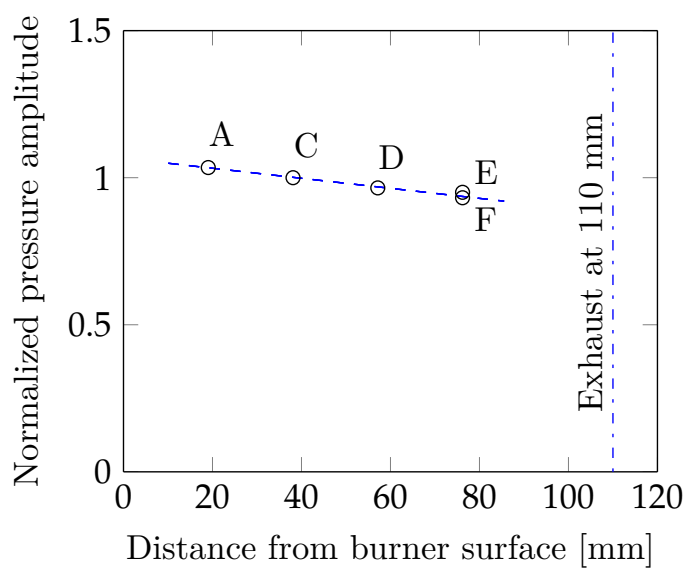

Figure B.2: Normalized pressure amplitude in combustion chamber w.r.t. point B 


\section{Single chamber Helmholtz analysis - extension of previous models}

The building block of the propsed reduced order model is the thermo-fluid analysis of a single Helmholtz resonator. This analysis is an extension from the model proposed by Hathout et al. [30].

Figure C.1 shows the schematics of single chamber Helmholtz resonator. Suppose we have a large volume "v" connected with an inlet and an outlet (labeled as "i" and "e" respectively). There is a mean flow flowing from inlet to outlet. Additionally, there are small bleeding holes (labeled as "b") on the walls (labeled as "w") of the volume, but the size of these holes are small so that the mass flow rate is much smaller than that of either inlet or exit. In a real combustor these bleed holes could be used to control the heat transfer and air dilution. Inside the volume there is heat source with an unsteady heat release rate of $\dot{Q}_{\mathrm{v}}$. There is also heat loss from the wall into the surroundings, whose rate is $\dot{Q}_{\mathrm{w}}$. The pressure of the upstream of the inlet is $P_{\mathrm{u}}$, the pressure of the downstream of the outlet is $P_{\mathrm{d}}$, and the pressure outside the bleeding holes is $P_{\mathrm{a}}$ ("a" stands for ambience). All components are axisymmetric, and it is assumed that all thermodynamic and fluid variables are uniform in each component. The meanings of the variables used below can be referenced to the nomenclature.

Applying the first law of thermodynamics to volume v, we have:

$$
V_{\mathrm{v}} \frac{d \rho_{\mathrm{v}} e_{\mathrm{v}}}{d t}=\dot{m}_{\mathrm{i}} h_{\mathrm{i}}-\dot{m}_{\mathrm{e}} h_{\mathrm{e}}-\dot{m}_{\mathrm{w}} h_{\mathrm{v}}+\dot{Q}_{\mathrm{v}}-\dot{Q}_{\mathrm{w}}
$$

If we neglect the temperature dependence of $c_{\mathrm{V}}$ and $c_{\mathrm{P}}$ for simplicity, then $e=c_{\mathrm{V}}\left(T-T_{\text {ref }}\right)$ and $h=c_{\mathrm{P}}\left(T-T_{\text {ref }}\right)$. Reference temperature $T_{\text {ref }}$ is taken as 0 here. Along with the relationships $c_{\mathrm{V}}=R /(\gamma-1), c_{\mathrm{P}}=R \gamma /(\gamma-1)$, and $P=\rho R T$, Eq.(C.1) can be written as:

$$
\frac{V_{\mathrm{v}}}{\gamma_{\mathrm{v}}-1} \frac{d P_{\mathrm{v}}}{d t}=\dot{m}_{\mathrm{i}} \frac{\gamma_{\mathrm{i}}}{\gamma_{\mathrm{i}}-1} R_{\mathrm{i}} T_{\mathrm{i}}-\dot{m}_{\mathrm{e}} \frac{\gamma_{\mathrm{e}}}{\gamma_{\mathrm{e}}-1} R_{\mathrm{e}} T_{\mathrm{e}}-\dot{m}_{\mathrm{w}} \frac{\gamma_{\mathrm{v}}}{\gamma_{\mathrm{v}}-1} R_{\mathrm{v}} T_{\mathrm{v}}+\dot{Q}_{\mathrm{v}}-\dot{Q}_{\mathrm{w}}
$$

Now substitute the speed of sound $C=\sqrt{\gamma R T}$ into Eq.(C.2) and assume $C$ to be constant, then take time derivative of Eq.(C.2), we will have:

$$
\frac{d^{2} P_{\mathrm{v}}}{d t^{2}}=\frac{\gamma_{\mathrm{v}}-1}{V_{\mathrm{v}}}\left(\frac{C_{\mathrm{i}}^{2}}{\gamma_{\mathrm{i}}-1} \frac{d \dot{m}_{\mathrm{i}}}{d t}-\frac{C_{\mathrm{e}}^{2}}{\gamma_{\mathrm{e}}-1} \frac{d \dot{m}_{\mathrm{e}}}{d t}\right)-\frac{C_{\mathrm{v}}^{2}}{V_{\mathrm{v}}} \frac{d \dot{m}_{\mathrm{w}}}{d t}+\frac{\gamma_{\mathrm{v}}-1}{V_{\mathrm{v}}}\left(\frac{d \dot{Q}_{\mathrm{v}}}{d t}-\frac{d \dot{Q}_{\mathrm{w}}}{d t}\right) .
$$

Equation (C.3) relates the pressure fluctuation inside the volume to the fluctuation of the mass flow rates in and out from the volume as well as the heat release and heat loss rate fluctuation. The fluctuation of mass flow rates in the inlet and outlet can be related to the velocity fluctuation inside them. The axial momentum equation inside the inlet can be written as:

$$
\rho_{\mathrm{i}} \frac{d U_{\mathrm{i}}}{d t}+\rho_{\mathrm{i}} U_{\mathrm{i}} \frac{d U_{\mathrm{i}}}{d x}=-\frac{d P_{\mathrm{i}}}{d x}+\nabla \cdot \sigma_{\mathrm{v}}
$$

As aforementioned, we assume that fluid mechanics variables are uniform in each component. If we assume a constant pressure gradient across the inlet and neglect surface shear stress, then Eq.(C.4) can be written as:

$$
\rho_{\mathrm{i}} \frac{d U_{\mathrm{i}}}{d t}=-\frac{d P_{\mathrm{i}}}{d x}=\frac{P_{\mathrm{u}}-P_{\mathrm{v}}}{l_{\mathrm{i}}} .
$$

Therefore the time derivative of mass flow rate through inlet can be obtained:

$$
\frac{d \dot{m}_{\mathrm{i}}}{d t}=A_{\mathrm{i}} \rho_{\mathrm{i}} \frac{d U_{\mathrm{i}}}{d t}=\frac{A_{\mathrm{i}}}{l_{\mathrm{i}}}\left(P_{\mathrm{u}}-P_{\mathrm{v}}\right)
$$


Similarly, the mass flow rate fluctuation inside outlet can be written as:

$$
\frac{d \dot{m}_{\mathrm{e}}}{d t}=\frac{A_{\mathrm{e}}}{l_{\mathrm{e}}}\left(P_{\mathrm{v}}-P_{\mathrm{d}}\right)
$$

The mass flow rates of bleeding from volume $\mathbf{v}$ depends on the specific configurations of the chamber. Assume that the size of the bleeding hole is a small fraction $\left(\epsilon_{\mathrm{A}}\right)$ of the total area of the walls of the volume $A_{\mathrm{w}}$ and the fluid inside the chamber is quiescent in radial direction, then the ideal discharge velocity through the bleeding holes is given by Bernoulli's equation as:

$$
\left|U_{\mathrm{w}}\right|=\sqrt{\frac{2\left|P_{\mathrm{v}}-P_{\mathrm{a}}\right|}{\rho_{\mathrm{v}}}} .
$$

Now taking the friction loss into consideration, the mass flow rate of the leakage is given by:

$$
\dot{m}_{\mathrm{w}}=\mathscr{C}_{\mathrm{d}} \epsilon_{\mathrm{A}} A_{\mathrm{w}} \rho_{\mathrm{v}} U_{\mathrm{w}}=\mathscr{C}_{\mathrm{d}} \epsilon_{\mathrm{A}} A_{\mathrm{w}} \sqrt{\frac{2 \rho_{\mathrm{v}}}{\left|P_{\mathrm{v}}-P_{\mathrm{a}}\right|}}\left(P_{\mathrm{v}}-P_{\mathrm{a}}\right),
$$

where $\mathscr{C}_{\mathrm{d}}$ is the discharge coefficient that depends on the shape and size of the hole and $\dot{m}_{\mathrm{w}}$ is positive when there is a net outflow. If the ambient conditions are steady, then taking the time derivative of Eq.(C.9) would yield:

$$
\frac{d \dot{m}_{\mathrm{w}}}{d t}=\mathscr{C}_{\mathrm{d}} \epsilon_{\mathrm{A}} A_{\mathrm{w}} \sqrt{\frac{\rho_{\mathrm{v}}}{2\left|P_{\mathrm{v}}-P_{\mathrm{a}}\right|}} \frac{d P_{\mathrm{v}}}{d t} .
$$

The physical process of heat loss through the wall consists of three parts: heat convection from the fluid inside the volume to the wall, heat conduction across the wall, and heat convection (as well as possible radiation) from the wall to the ambience. The heat loss $\dot{Q}_{\mathrm{w}}$ can be analyzed through a thermal circuit analysis:

$$
\dot{Q}_{\mathrm{w}}=\frac{T_{\mathrm{v}}-T_{\mathrm{a}}}{\Omega},
$$

where $\Omega$ is the total thermal resistance related to the heat conduction coefficient $(\kappa)$, heat convection coefficient $(\eta)$ and heat radiation. The specific form of $\Omega$ depends on the configuration of the resonator in study. Again, if we assume the ambient conditions are steady, then the time derivative of Eq.(C.11) takes the form:

$$
\frac{d \dot{Q}_{\mathrm{w}}}{d t}=\frac{1}{\Omega} \frac{d T_{\mathrm{v}}}{d t}=\frac{1}{\Omega \rho_{\mathrm{v}} R_{\mathrm{v}}} \frac{d P_{\mathrm{v}}}{d t} .
$$

Now if we substitute Eqs.(C.6), (C.7), (C.10), and (C.12) into Eq.(C.3), separate $V_{\mathrm{v}}$ into $V_{\mathrm{v}}=A_{\mathrm{v}} l_{\mathrm{v}}$ and rearrange terms in Eq.(C.3), we will have:

$$
\frac{d^{2} P_{\mathrm{v}}}{d t^{2}}+\left(\mathscr{T}_{\mathrm{i}}+\mathscr{T}_{\mathrm{ii}}\right) \frac{d P_{\mathrm{v}}}{d t}+\left(\mathscr{T}_{\mathrm{iii}}+\mathscr{T}_{\mathrm{iv}}\right) P_{\mathrm{v}}=\mathscr{T}_{\mathrm{iii}} P_{\mathrm{u}}+\mathscr{T}_{\mathrm{iv}} P_{\mathrm{d}}+\frac{\gamma_{\mathrm{v}}-1}{V_{\mathrm{v}}} \frac{d \dot{Q}_{\mathrm{v}}}{d t},
$$

where the terms $\mathscr{T}_{\text {n }}$ are:

$$
\begin{gathered}
\mathscr{T}_{\mathrm{i}}=\frac{C_{\mathrm{v}}^{2}}{V_{\mathrm{v}}} \mathscr{C}_{\mathrm{d}} \epsilon_{\mathrm{A}} A_{\mathrm{w}} \sqrt{\frac{2 \rho_{\mathrm{v}}}{\left|P_{\mathrm{v}}-P_{\mathrm{a}}\right|},} \\
\mathscr{T}_{\mathrm{ii}}=\frac{\gamma_{\mathrm{v}}-1}{V_{\mathrm{v}}} \frac{1}{\Omega \rho_{\mathrm{v}} R_{\mathrm{v}}}, \\
\mathscr{T}_{\mathrm{iii}}=\frac{\gamma_{\mathrm{v}}-1}{\gamma_{\mathrm{i}}-1}\left(\frac{C_{\mathrm{i}}^{2} A_{\mathrm{i}}}{V_{\mathrm{v}} l_{\mathrm{i}}}\right), \\
\mathscr{T}_{\mathrm{iv}}=\frac{\gamma_{\mathrm{v}}-1}{\gamma_{\mathrm{e}}-1}\left(\frac{C_{\mathrm{e}}^{2} A_{\mathrm{e}}}{V_{\mathrm{v}} l_{\mathrm{e}}}\right) .
\end{gathered}
$$


Equation C.13 is the pressure governing equation for a single chamber Helmholtz resonator with different inlet/outlet temperature and gas composition. It also considers the phenomena of fluid bleeding and wall heat transfer. From it we can also assess the contributions of different heat/mass transfer to the system dynamics. Specifically, fluid leakage through bleeding holes (term $\mathscr{T}_{\mathrm{i}}$ ) and wall heat loss (term $\mathscr{T}_{\mathrm{ii}}$ ) provide damping to the system. The geometry of the inlet and outlet (term $\mathscr{T}_{\text {iii }}$ and $\mathscr{T}_{\text {iv }}$ ) affects the system resonance frequency. And the driving force of the system is provided by a combination of upstream pressure $P_{\mathrm{u}}$, downstream pressure $P_{\mathrm{d}}$, and internal heat release rate $\dot{Q}_{\mathrm{V}}$. 


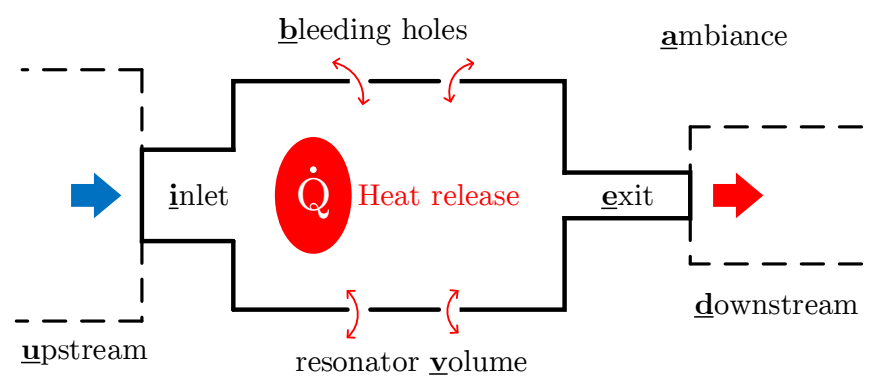

Figure C.1: Schematic of a single chamber Helmholtz resonator 


\section{Modeling of heat transfer across the combustor walls}

In the current application of the proposed model on the GTMC, we consider two parallel paths of heat loss across the combustor wall.

The first path represent the heat transfer from the fluid through the combustion chamber wall into the ambience. We dente the thermal resistance through the first path as $\Omega_{\mathrm{i}} . \Omega_{\mathrm{i}}$ is determined by the combination of fluid to wall convection coefficient $\eta_{2 \mathrm{w}}$, wall conduction coefficient $\kappa_{\mathrm{w}}$, wall to ambience convection coefficient $\eta_{\mathrm{wa}}$, as well as wall area $A_{\mathrm{w}}$ and thickness $l_{\mathrm{w}}$ :

$$
\Omega_{\mathrm{i}}=\frac{1}{\eta_{2 \mathrm{w}} A_{\mathrm{w}}}+\frac{l_{\mathrm{w}}}{\kappa_{\mathrm{w}} A_{\mathrm{w}}}+\frac{1}{\eta_{\mathrm{wa}} A_{\mathrm{w}}} .
$$

In convection heat transfer analysis it is convenient to represent the heat transfer coefficient $\eta$ in terms of Nusselt number $(N u=\eta l / \kappa)$, where $l$ is the characteristic length and $\kappa$ is the heat conduction coefficient of the fluid evaluated at the mean temperature between fluid and wall. Heat transfer from fluid to wall is a forced convection process, the mean Nusselt number for a turbulent flow through a flat plate $\left(\overline{N u}_{\mathrm{fc}}\right)$ is [41]:

$$
\overline{N u}_{\mathrm{fc}}=0.037 \operatorname{Re}^{4 / 5} \operatorname{Pr}^{1 / 3} \text {, }
$$

where $R e=U l / v$ is the Reynolds number defined based on the plate length and $P r=v / \alpha$ is the Prandtl number evaluated at the mean temperature at the mean temperature between fluid and wall.

On the other hand, heat transfer from wall to ambience is a natural convection process. The Nusselt number for a natural convection through a vertical plate with a turbulent boundary layer $\left(\overline{N u}_{\mathrm{nt}}\right)$ can be calculated by [48]:

$$
\overline{N u}_{\mathrm{nt}}=\left\{0.825+\frac{0.387 R a^{1 / 6}}{\left[1+(0.492 / P r)^{9 / 16}\right]^{8 / 27}}\right\}^{2},
$$

where $R a=g\left(T_{\mathrm{w}}-T_{\mathrm{a}}\right) l^{3} /\left(v \alpha T_{\mathrm{wa}}\right)$ is the Rayleigh number, $g$ is the gravitational constant, $T_{\mathrm{wa}}$ is the mean temperature between the wall and the ambience. With these formulations, the convection heat transfer coefficients $\eta_{2 \mathrm{w}}$ and $\eta_{\mathrm{wa}}$ can be calculated by $\eta_{2 \mathrm{w}}=\bar{N}_{\mathrm{fc}} \kappa_{2 \mathrm{w}} / l_{2}$ and $\eta_{\mathrm{wa}}=\overline{N u}_{\mathrm{nt}} \kappa_{\mathrm{wa}} / l_{2}$ respectively. Hence $\Omega_{\mathrm{i}}$ can be calculated from Eq.(D.1).

The second path is the direct radiation from the fluid inside the combustion chamber into the ambience, and its thermal resistance is denoted as $\Omega_{\mathrm{ii}}$. Since $\Omega_{\mathrm{ii}}$ represent a single radiation process, it is simply the inverse of the product of radiation heat transfer coefficient and wall area:

$$
\Omega_{\mathrm{ii}}=\frac{1}{A_{\mathrm{w}} \cdot \epsilon_{\mathrm{e}} \sigma_{\mathrm{S}-\mathrm{B}}\left(T_{2}^{2}+T_{0}^{2}\right)\left(T_{2}+T_{0}\right)},
$$

where $\epsilon_{\mathrm{e}}$ is the emissivity of the fluid and $\sigma_{\mathrm{S}-\mathrm{B}}$ is the Stefan-Boltzmann constant $\left(\sigma_{\mathrm{S}-\mathrm{B}}=5.67 \times 10^{-8} \mathrm{~W} / \mathrm{m}^{2} \cdot \mathrm{K}^{4}\right)$.

With these analysis, the total thermal resistance across the combustor walls, $\Omega$, can be determined through heat circuit analysis [41] as:

$$
\frac{1}{\Omega}=\frac{\dot{Q}_{\mathrm{w}}}{T_{2}-T_{0}}=\frac{1}{\Omega_{\mathrm{i}}}+\frac{1}{\Omega_{\mathrm{ii}}}
$$




\section{References}

[1] Rayleigh, J. W. S., "The Explanation of Certain Acoustical Phenomena," Nature, Vol. 18, No. 455, July 1878, pp. 319-321.

[2] Zinn, B. T. and Lieuwen, T. C., "Combustion Instabilities: Basic Concepts," Lieuwen and Yang [49], chap. 1.

[3] Mongia, H. C., Held, T. J., Hsiao, G. C., and Pandalai, R. P., "Challenges and Progress in Controlling Dynamics in Gas Turbine Combustors," Journal of Propulsion and Power, Vol. 19, No. 5, 2003, pp. 822-829.

[4] Lieuwen, T. C. and Zinn, B. T., "The role of equivalence ratio oscillations in driving combustion instabilities in low NOx gas turbines," Symposium (International) on Combustion, Vol. 27, No. 2, Jan. 1998, pp. 1809-1816.

[5] Schuller, T., Durox, D., and Candel, S. M., "Dynamics of and noise radiated by a perturbed impinging premixed jet flame," Combustion and Flame, Vol. 128, No. 1-2, Jan. 2002, pp. 88-110.

[6] Krebs, W., Bethke, S., Lepers, J., Flohr, P., Prade, B., Johnson, C., and Sattinger, S., "Thermoacoustic Design Tools and Passive Control: Siemens Power Generation Approaches," Lieuwen and Yang [49], chap. 5.

[7] Cohen, J. M. and Banaszuk, A., "Factors Affecting the Control of Unstable Combustors," Journal of Propulsion and Power, Vol. 19, No. 5, 2003, pp. 811-821.

[8] Cohen, J. M., Rey, N. M., Jacobson, C. A., and Anderson, T. J., "Active Control of Combustion Instability in a Liquid-Fueled Low-NOx Combustor," Journal of Engineering for Gas Turbines and Power, Vol. 121, No. 2, 1999, pp. 281.

[9] Temme, J. E., Allison, P. M., and Driscoll, J. F., "Combustion instability of a lean premixed prevaporized gas turbine combustor studied using phase-averaged PIV," Combustion and Flame, Vol. 161, No. 4, April 2014, pp. 958-970.

[10] Zähringer, K., Durox, D., and Lacas, F., "Helmholtz behavior and transfer function of an industrial fuel swirl burner used in heating systems," International Journal of Heat and Mass Transfer, Vol. 46, No. 18, 2003, pp. 3539-3548.

[11] Uhm, J. H. and Acharya, S., "Low-bandwidth open-loop control of combustion instability," Combustion and Flame, Vol. 142, No. 4, 2005, pp. 348-363.

[12] Durox, D., Schuller, T., Noiray, N., Birbaud, a. L., and Candel, S. M., "Rayleigh criterion and acoustic energy balance in unconfined self-sustained oscillating flames," Combustion and Flame, Vol. 156, No. 1, 2009, pp. 106-119.

[13] McManus, K. R., Poinsot, T., and Candel, S. M., "A review of active control of combustion instabilities," Progress in Energy and Combustion Science, Vol. 19, No. 1, 1993, pp. 1-29.

[14] Ducruix, S., Schuller, T., Durox, D., and Candel, S. M., "Combustion Dynamics and Instabilities: Elementary Coupling and Driving Mechanisms," Journal of Propulsion and Power, Vol. 19, No. 5, Sept. 2003, pp. 722-734. 
[15] Weigand, P., Meier, W., Duan, X. R., Stricker, W., and Aigner, M., "Investigations of swirl flames in a gas turbine model combustor I. Flow field, structures, temperature, and species distributions," Combustion and Flame, Vol. 144, No. 1-2, Jan. 2006, pp. 205-224.

[16] Giezendanner-Thoben, R., Keck, O., Weigand, P., Meier, W., Meier, U., Stricker, W., and Aigner, M., "Periodic combustion instabilities in a swirl burner studied by phase-locked planar laser-induced fluorescence," Combustion Science and Technology, Vol. 175, 2003, pp. 721-741.

[17] Giezendanner-Thoben, R., Weigand, P., Duan, X. R., Meier, W., Meier, U., Aigner, M., and Lehmann, B., "Laser-Based Investigations of Periodic Combustion Instabilities in a Gas Turbine Model Combustor," Journal of Engineering for Gas Turbines and Power, Vol. 127, No. 3, 2005, pp. 492-496.

[18] Steinberg, A. M., Boxx, I., Stöhr, M., Meier, W., and Carter, C. D., "Effects of Flow Structure Dynamics on Thermoacoustic Instabilities in Swirl-Stabilized Combustion," AIAA Journal, Vol. 50, No. 4, April 2012, pp. 952-967.

[19] Stöhr, M., Arndt, C. M., and Meier, W., "Effects of Damköhler number on vortex-flame interaction in a gas turbine model combustor," Proceedings of the Combustion Institute, Vol. 34, No. 2, Jan. 2013, pp. 3107-3115.

[20] Steinberg, A. M., Boxx, I., Stöhr, M., Carter, C. D., and Meier, W., "Flow-flame interactions causing acoustically coupled heat release fluctuations in a thermo-acoustically unstable gas turbine model combustor," Combustion and Flame, Vol. 157, No. 12, Dec. 2010, pp. 2250-2266.

[21] Duan, X. R., Meier, W., Weigand, P., and Lehmann, B., "Phase-resolved laser Raman scattering and laser Doppler velocimetry applied to periodic instabilities in a gas turbine model combustor," Applied Physics B, Vol. 80, No. 3, Jan. 2005, pp. 389-396.

[22] Stöhr, M., Boxx, I., Carter, C. D., and Meier, W., "Experimental study of vortex-flame interaction in a gas turbine model combustor," Combustion and Flame, Vol. 159, No. 8, Aug. 2012, pp. 2636-2649.

[23] Stöhr, M., Arndt, C. M., and Meier, W., "Transient effects of fuel-air mixing in a partially-premixed turbulent swirl flame," Proceedings of the Combustion Institute, Vol. 35, No. 3, July 2015, pp. 3327-3335.

[24] Stöhr, M., Sadanandan, R., and Meier, W., "Phase-resolved characterization of vortex-flame interaction in a turbulent swirl flame," Experiments in Fluids, Vol. 51, No. 4, June 2011, pp. 1153-1167.

[25] Meier, W., Duan, X. R., and Weigand, P., "Investigations of swirl flames in a gas turbine model combustor II. Turbulence-chemistry interactions," Combustion and Flame, Vol. 144, No. 1-2, Jan. 2006, pp. 225-236.

[26] Allison, P. M., Driscoll, J. F., and Ihme, M., "Acoustic characterization of a partially-premixed gas turbine model combustor: Syngas and hydrocarbon fuel comparisons," Proceedings of the Combustion Institute, Vol. 34, No. 2, Jan. 2013, pp. 3145-3153.

[27] Allison, P. M., Chen, Y. T., and Driscoll, J. F., "Investigation of Dimethyl Ether Combustion Instabilities in a Partially - Premixed Gas Turbine Model Combustor Using High-Speed Laser Diagnostics," AIAA Aerospace 
[28] Allison, P. M., Chen, Y. T., Ihme, M., and Driscoll, J. F., "Coupling of flame geometry and combustion instabilities based on kilohertz formaldehyde PLIF measurements," Proceedings of the Combustion Institute, Vol. 35, No. 3, July 2015, pp. 3255-3262.

[29] See, Y. C. and Ihme, M., "Large eddy simulation of a partially-premixed gas turbine model combustor," Proceedings of the Combustion Institute, Vol. 35, No. 2, Sept. 2015, pp. 1225-1234.

[30] Hathout, J. P., Fleifil, M., Annaswamy, A. M., and Ghoniem, A. F., "Combustion Instability Active Control Using Periodic Fuel Injection," Journal of Propulsion and Power, Vol. 18, No. 2, March 2002, pp. 390-399.

[31] Candel, S. M., Durox, D., Schuller, T., Bourgouin, J.-F., and Moeck, J. P., "Dynamics of Swirling Flames," Annual Review of Fluid Mechanics, Vol. 46, No. 1, Jan. 2014, pp. 147-173.

[32] Noiray, N., Durox, D., Schuller, T., and Candel, S. M., "A unified framework for nonlinear combustion instability analysis based on the flame describing function," Journal of Fluid Mechanics, Vol. 615, Nov. 2008, pp. 139-167.

[33] Schuller, T., Durox, D., Palies, P., and Candel, S. M., "Acoustic decoupling of longitudinal modes in generic combustion systems," Combustion and Flame, Vol. 159, No. 5, May 2012, pp. 1921-1931.

[34] Palies, P., Durox, D., Schuller, T., and Candel, S. M., "Nonlinear combustion instability analysis based on the flame describing function applied to turbulent premixed swirling flames," Combustion and Flame, Vol. 158, No. 10, Oct. 2011, pp. 1980-1991.

[35] Silva, C. F., Nicoud, F., Schuller, T., Durox, D., and Candel, S. M., "Combining a Helmholtz solver with the flame describing function to assess combustion instability in a premixed swirled combustor," Combustion and Flame, Vol. 160, No. 9, Sept. 2013, pp. 1743-1754.

[36] Chen, Y. T. and Driscoll, J. F., "Experimental Studies and Modeling of Acoustic Instabilities in a Gas Turbine Model Combustor," AIAA Aerospace Sciences Meeting, American Institute of Aeronautics and Astronautics, Kissimmee, FL, Jan. 2015, AIAA paper 2015-1566.

[37] Rosenberg, D. A., Allison, P. M., and Driscoll, J. F., "Flame index and its statistical properties measured to understand partially premixed turbulent combustion," Combustion and Flame, Vol. 162, No. 7, July 2015, pp. 2808-2822.

[38] Samaniego, J.-M., Egolfopoulos, F. N., and Bowman, C. T., "CO2* Chemiluminescence in Premixed Flames," Combustion Science and Technology, Vol. 109, No. 1-6, 1995, pp. 183-203.

[39] Lee, J. G. and Santavicca, D. A., "Experimental Diagnostics for the Study of Combustion Instabilities in Lean Premixed Combustors," Journal of Propulsion and Power, Vol. 19, No. 5, 2003, pp. 735-750.

[40] Ayoola, B. O., Balachandran, R., Frank, J. H., Mastorakos, E., and Kaminski, C. F., "Spatially resolved heat release rate measurements in turbulent premixed flames," Combustion and Flame, Vol. 144, No. 1-2, 2006, pp. 1-16. 
[41] Incropera, F. P., Dewitt, D. P., Bergman, T. L., and Lavine, A. S., Fundamentals of Heat and Mass Transfer, Wiley, 2007.

[42] Tennekes, H. and Lumley, J. L., A First Course in Turbulence, MIT Press, 1972.

[43] Arcoumanis, C., Bae, C., Crookes, R., and Kinoshita, E., "The potential of di-methyl ether (DME) as an alternative fuel for compression-ignition engines: A review," Fuel, Vol. 87, No. 7, June 2008, pp. 1014-1030.

[44] Durox, D., Schuller, T., and Candel, S. M., "Self-induced instability of a premixed jet flame impinging on a plate," Proceedings of the Combustion Institute, Vol. 29, Jan. 2002, pp. 69-75.

[45] Brackmann, C., Nygren, J., Bai, X., Li, Z., Bladh, H., Axelsson, B., Denbratt, I., Koopmans, L., Bengtsson, P.-E., and Aldén, M., "Laser-induced fluorescence of formaldehyde in combustion using third harmonic Nd:YAG laser excitation," Spectrochimica Acta Part A: Molecular and Biomolecular Spectroscopy, Vol. 59, No. 14, Dec. 2003, pp. 3347-3356.

[46] Yu, K. H., Trouvé, A., and Daily, J. W., "Low-frequency pressure oscillations in a model ramjet combustor," Journal of Fluid Mechanics, Vol. 232, April 1991, pp. 47-72.

[47] Ahlborn, B., Seto, M. L., and Noack, B. R., "On drag, Strouhal number and vortex-street structure," Fluid Dynamics Research, Vol. 30, No. 6, June 2002, pp. 379-399.

[48] Churchill, S. W. and Chu, H. H., "Correlating equations for laminar and turbulent free convection from a vertical plate," International Journal of Heat and Mass Transfer, Vol. 18, No. 11, 1975, pp. 1323 - 1329.

[49] Lieuwen, T. C. and Yang, V., editors, Combustion Instabilities in Gas Turbine Engines, Vol. 210 of Progress in Astronautics and Aeronautics, AIAA, 2005. 